\title{
Channel, tube, and Taylor-Couette flow of complex viscoelastic fluid models
}

\section{Journal Article}

\section{Author(s):}

Dressler, M.; Edwards, B.J.

Publication date:

2006-10

Permanent link:

https://doi.org/10.3929/ethz-b-000035639

Rights / license:

In Copyright - Non-Commercial Use Permitted

Originally published in:

Rheologica Acta 46(1), https://doi.org/10.1007/s00397-006-0085-3 


\section{Dressler \\ B. J. Edwards}

\section{Channel, tube, and Taylor-Couette flow of complex viscoelastic fluid models}

Received: 19 December 2005

Accepted: 12 January 2006

Published online: 24 May 2006

(C) Springer-Verlag 2006

\author{
M. Dressler $(\bowtie)$ \\ Laboratory of Food Process \\ Engineering, Eidgenössische \\ Technische Hochschule (ETH) Zürich, \\ CH-8092 Zürich, Switzerland \\ e-mail: marco.dressler@ilw.agrl.ethz.ch \\ Tel.: +41-1-6325340 \\ Fax: +41-1-6321155 \\ B. J. Edwards \\ Department of Chemical Engineering, \\ University of Tennessee, \\ Knoxville, TN 37996, USA
}

\begin{abstract}
We show how to formulate two-point boundary value problems to compute laminar channel, tube, and Taylor-Couette flow profiles for some complex viscoelastic fluid models of differential type. The models examined herein are the Pom-Pom Model [McLeish and Larson 42:81-110, (1998)] the Pompon Model [Öttinger 40:317-321, (2001)] and the Two Coupled Maxwell Modes Model (Beris and Edwards 1994). For the two-mode Upper-Convected Maxwell Model, we calculate analytical solutions for the three flow geometries and use the solutions to validate the numerical methodology. We illustrate how to calculate the velocity, pressure, conformation tensor, backbone orientation tensor, backbone stretch, and extra stress profiles for various mod-
\end{abstract}

els. For the Pom-Pom Model, we find that the two-point boundary value problem is numerically unstable, which is due to the aphysical nonmonotonic shear stress vs shear rate prediction of the model. For the other two models, we compute laminar flow profiles over a wide range of pressure drops and inner cylinder velocities. The volumetric flow rate and the nonlinear viscoelastic material properties on the boundaries of the flow geometries are determined as functions of the applied pressure drop, allowing easy analysis of experimentally measurable quantities.

Keywords Differential constitutive equations · Polymer melts · Laminar flows $\cdot$ Maxwell models Pom-Pom Model

\section{Introduction}

The flow of non-Newtonian liquids through channels, tubes, and in the Taylor-Couette geometry (i.e., concentric cylinders) is encountered in many industrial applications involving plastics, food, and extraction of raw materials. It is, therefore, of interest in applied and basic research in the areas of engineering and physics. In capillary rheometry, e.g., the nonlinear rheological properties of complex fluids are measured indirectly via the volumetric flow rate through the capillary. From the volumetric flow rate and the pressure drop, one determines the wall shear rate and the shear viscosity. However, for viscoelastic liquids, the relationship between wall shear rate, volumetric flow rate, and extra stress is more complicated than for Newtonian liquids due to the highly nonlinear viscometric material functions and the normal stresses. Taylor-Couette flows are encountered in many applications, such as the journal bearing, and they are modeled as annular flows between concentric or eccentric cylinders. The fluid dynamics here are even more complicated than in channel or tube flows, as there can exist non-Newtonian flow behavior and centrifugal forces due to rotation of the inner and/or outer cylinder.

To describe these flows theoretically for non-Newtonian materials, it is necessary to use a constitutive model for relating the flow kinematics to the fluid's microstructure and internal state of tension. Many models exist for this purpose, and those that produce the best quantitative descriptions and predictions of viscoelastic fluid responses are quite complex internally. These more descriptive models employ multiple microstructural variables subject to coupled evolution equations, which relate changes in the fluid's microstructure 
to the flow kinematics. In the past 10 years, the complexity of viscoelastic fluid models has increased in direct proportion with their descriptive capabilities.

In the 1980s, much effort was expended to develop finite element methods (FEMs) to solve viscoelastic fluid models; however, the simpler models available during that era were not very successful at describing real material behavior. Now that much better models are available, it is natural to try to apply finite-element methodology to these new models, but this is not as straightforward as it seems: the newer, better models are much more complicated than the models used in the 1980s. Consequently, they are in practice, although not necessarily in principle, harder to implement using finite element techniques.

As a consequence, it would be beneficial to have a simple technique available that could be used to obtain relevant information from complex models in flow fields of practical importance. For example, a valid question for an experimentalist to ask might be: "What are the wall shear rate and wall shear stress of a fluid following the Pom-Pom Model as functions of the flow rate in Poiseuille flow?" Developing an FEM to solve this problem presents several challenges, including coding difficulties, which limit the use of these more complicated models in inhomogeneous flow calculations.

Even the most complicated viscoelastic fluid model is easy to solve in homogeneous flow calculations, using techniques such as the Newton-Raphson Method (for steady-state flows) or the Runge-KuttaMethod (for transient flows). It would be a boon to have simple techniques available for solving these complex models in inhomogeneous flows as well. Such a technique would require not much more time than homogeneous flow calculations, which run virtually instantaneously on contemporary personal computers. Of course, with simplicity comes a trade-off between ease of use and exactly what can and cannot be described with the simpler methodology. Fortunately, the needs of most rheologists are not that demanding: being able to address the question presented in the preceding paragraph without too much difficulty would be a nice advance.

In the present article, we want to solve several of the more complicated viscoelastic fluid models of differential type for non-homogeneous shearing flows as they are encountered in several important geometries, such as the rectangular channel, circular tube, and the Taylor-Couette geometry. We will consider in this article the Pom-Pom Model (McLeish and Larson 1998) of branched-chain macromolecules, the thermodynamically consistent Pompon Model (Öttinger 2001), and the Two Coupled Maxwell Modes (TCMM) Model (Beris and Edwards 1994; Edwards et al. 1996). This will allow us to discuss with these examples the complicated relationships between inertia, nonlinear flow profiles, viscoelastic stresses, wall viscometric material functions, and volumetric flow rates in inhomogeneous flows of practical importance. These examples serve to illustrate the complications and difficulties encountered in the experimental characterization and theoretical description of non-Newtonian fluids.

There are several reasons for using the above-stated three models in the present article. First, these models have not yet been widely used in inhomogeneous flow calculations, primarily because of their inherent complexity. The second reason is because the models are inherently complex: our motivation for this paper is to illustrate how complicated models can be solved in inhomogeneous flows with no more difficulty than simple models in homogeneous flows. Consequently, we should use complicated models in our illustrations rather than simple ones. These models have all been used recently for fairly accurate quantitative descriptions of rheological behavior for homogeneous flow fields (Chodankar et al. 2003; Edwards et al. 2002; Jiang et al. 2003; Kamerkar et al. 2005); in this study, we extend this work for inhomogeneous flow fields.

Much work has been done in the past to solve simpler rheological models for one-dimensional inhomogeneous flows. The Giesekus Model (Giesekus 1982) has been solved by Schleiniger and Weinacht (1991) for steady Poiseuille flow through channels and tubes. The PhanThien-Tanner Model (Phan-Thien and Tanner 1977) has been solved analytically and numerically for a number of non-homogeneous shear flows in various flow geometries (e.g., in Oliveira and Pinho 1999; Pinho and Oliveira 2000a,b; Alves et al. 2001; Cruz and Pinho 2004). In Dressler and Edwards (2005), it has been shown how viscoelastic fluid models for polymer blends can be reformulated in terms of two-point boundary value (TPBV) problems to solve for laminar channel flow. The method is essentially a transformation of a set of partial differential equations (PDEs) in terms of a set of ordinary differential equations (ODEs) for the non-trivial spatial coordinate.

In the present article, the Pom-Pom, Pompon, and TCMM models will be solved for laminar channel, tube, and TaylorCouette flow (with and without axial through-flow) using the methodology proposed in Dressler and Edwards (2005). We determine the nonlinear flow profiles, extra stress tensor profiles, backbone stretch profiles, and the wall viscometric material properties in the laminar flow regime for the three models.

The article is organized as follows. First, the three viscoelastic fluid models are introduced. Then, the three sets of model equations are reformulated for the laminar channel and tube flow problems and it is explained how the model equations can be reformulated in terms of a TPBV problem to solve for mixed Poiseuille and Couette flow. Analytical solutions will be derived for the Two-Mode Upper Convected Maxwell (2MUCM) Model in channel and tube flow. Then, we report and compare sample calculations for the three viscoelastic fluid models. We find that the PomPom Model predicts an aphysical non-monotonic wall shear stress vs shear rate relationship, exactly analogous to its 
aphysical prediction in homogeneous steady-shear flow. In the second part of the article, the methodology is then applied to the Pompon and TCMM models to solve for laminar flow in the Taylor-Couette geometry with axial through-flow.

\section{Model equations}

The Pompon Model (Öttinger 2001) is a thermodynamically consistent reformulation of the Pom-Pom Model (McLeish and Larson 1998) that accounts for, among other things, a non-trivial second normal stress coefficient in shear flow. For this model, the physical variables are the mass density, $\rho$, the momentum density, $\mathbf{M}=\rho \mathbf{v}$ ( $\mathbf{v}$ being the velocity field), the contravariant backbone orientation tensor, $\mathbf{S}$, and the scalar backbone stretch, $\lambda$. The time evolution equations of the Pompon Model are

$\frac{\partial \rho}{\partial t}=-\nabla \cdot(\mathbf{v} \rho)$,

$\rho \frac{\partial \mathbf{v}}{\partial t}=-\rho \mathbf{v} \cdot \nabla \mathbf{v}-\nabla p+\nabla \cdot \boldsymbol{\sigma}$,

$$
\begin{aligned}
\frac{\partial \mathbf{S}}{\partial t}= & -\mathbf{v} \cdot \nabla \mathbf{S}+\mathbf{S} \cdot \mathbf{L}^{\mathrm{T}}+\mathbf{L} \cdot \mathbf{S} \\
& -\frac{1}{\left(1+a_{1}\right) \lambda_{\mathrm{b}}}\left\{3 \mathbf{S} \cdot \mathbf{S}+\left(a_{1}-1\right) \mathbf{S}-\frac{a_{1}}{3} \mathbf{1}\right\},
\end{aligned}
$$

$$
\frac{\partial \lambda}{\partial t}=-\mathbf{v} \cdot \boldsymbol{\nabla} \lambda+\lambda \mathbf{L}: \mathbf{S}-\frac{1}{\lambda_{\mathrm{s}}}(\lambda-\mathbf{1}) e^{\nu^{*}(\lambda-1)},
$$

where $\partial \cdot / \partial t$ and $\nabla$ denote partial derivatives with respect to time, $t$, and space, $\mathbf{r}$, respectively, and $\mathbf{L}^{\mathrm{T}}=\nabla \mathbf{v}$ is the velocity gradient tensor. We adopt the Gibbs notation here as we work in terms of Cartesian and cylindrical coordinates in the present article. Equation 1 is the continuity equation for the mass density, $\rho$, and Eq. 2 is the Cauchy momentum balance equation for the velocity field, $\mathbf{v}$, where the pressure and the extra stress tensor have been denoted with $p$ and $\sigma$, respectively.

The first term on the right side of Eq. 2 is the nonlinear convective term, the second one represents the negative pressure gradient, and the last one is the divergence of the extra stress tensor field given by Eq. 5 below. Equation 3 is the time evolution equation for the backbone orientation tensor as reported by Chodankar et al. (2003) (Eq. 6), where $\lambda_{\mathrm{b}}$ is a relaxation time and $a_{1}$ is a phenomenological parameter which is determined by the ratio of normal stress differences in the zero shear limit, $a_{1}=-\left(1+N_{1} /\left(2 N_{2}\right)\right)$.
Equation 4 is the time evolution equation for the backbone stretch, where $\lambda_{\mathrm{s}}$ is a second relaxation time and the parameter $\nu^{*}$ is related to the number of arms, $q$, of the Pompon molecule: $\nu^{*}=2 / q$. The expression for the extra stress tensor that renders the Cauchy momentum balance thermodynamically consistent with the microstructural Eqs. 3 and 4 is

$\boldsymbol{\sigma}=C g \lambda^{2} \mathbf{S}$,

where $C=15 / 4$ and $g$ is the elastic modulus of the fluid. Equations 1, 2, 3, 4 and 5 constitute a set of PDEs to describe the macroscopic dynamics of a Pompon fluid, and it corresponds to the equations solved in Chodankar et al. (2003) for double step-strain flows.

In the Pom-Pom Model (McLeish and Larson 1998), the backbone orientation dynamics are described in terms of a second-rank contravariant tensor, $\mathbf{A}$, which is related to the backbone orientation tensor, $\mathbf{S}=\mathbf{A} / \operatorname{tr} \mathbf{A}$. The time evolution equation of the tensor, $\mathbf{A}$, according to Chodankar et al. (2003) (Eq. 5) reads

$\frac{\partial \mathbf{A}}{\partial t}=-\mathbf{v} \cdot \boldsymbol{\nabla} \mathbf{A}+\mathbf{A} \cdot \mathbf{L}^{\mathrm{T}}+\mathbf{L} \cdot \mathbf{A}-\frac{1}{\lambda_{\mathrm{b}}}(\mathbf{A}-\mathbf{1})$,

and it substitutes Eq. 3 in the set of Pompon Eqs. 1, 2, 3 and 4 to yield the Pom-Pom Model. Note that the Pom-Pom Model has not been derived rigorously from nonequilibrium thermodynamics. The implications of this will be discussed below.

For the TCMM Model (Beris and Edwards 1994; Edwards et al. 1996), the microstructural variables of the fluid are two contravariant second-rank conformation tensors, $\mathbf{C}^{i}(i=\{1,2\})$. The model equations consist of the continuity (Eq. 1) and the Cauchy momentum (Eq. 2) for the mass density and the velocity field, respectively. For the time evolution equations of the microstructural variables, the equations of change are

$$
\begin{aligned}
\frac{\partial \mathbf{C}^{1}}{\partial t}= & -\mathbf{v} \cdot \nabla \mathbf{C}^{1}+\mathbf{C}^{1} \cdot \mathbf{L}^{\mathrm{T}}+\mathbf{L} \cdot \mathbf{C}^{1}-\frac{1}{\lambda_{1}} \mathbf{C}^{1} \\
& +\frac{k_{\mathrm{B}} T}{\lambda_{1} K_{1}} \mathbf{1}-\frac{\theta}{2 k_{\mathrm{B}} T} \sqrt{\frac{n_{2}}{n_{1}}} \frac{1}{\sqrt{\lambda_{1} \lambda_{2}}} \\
& \times\left[K_{2}\left(\mathbf{C}^{1} \cdot \mathbf{C}^{2}+\mathbf{C}^{2} \cdot \mathbf{C}^{1}\right)-2 k_{\mathrm{B}} T \mathbf{C}^{1}\right], \\
\frac{\partial \mathbf{C}^{2}}{\partial t}= & -\mathbf{v} \cdot \boldsymbol{\nabla} \mathbf{C}^{2}+\mathbf{C}^{2} \cdot \mathbf{L}^{\mathrm{T}}+\mathbf{L} \cdot \mathbf{C}^{2}-\frac{1}{\lambda_{2}} \mathbf{C}^{2} \\
& +\frac{k_{\mathrm{B}} T}{\lambda_{2} K_{2}} \mathbf{1}-\frac{\theta}{2 k_{\mathrm{B}} T} \sqrt{\frac{n_{1}}{n_{2}}} \frac{1}{\sqrt{\lambda_{1} \lambda_{2}}} \\
& \times\left[K_{1}\left(\mathbf{C}^{1} \cdot \mathbf{C}^{2}+\mathbf{C}^{2} \cdot \mathbf{C}^{1}\right)-2 k_{\mathrm{B}} T \mathbf{C}^{2}\right] .
\end{aligned}
$$


In the above equations, the quantities $\lambda_{i}, n_{i}$, and $K_{i}$ are the relaxation time, the number density of microstructural units per unit volume, and the characteristic elastic constants belonging to conformation tensor $\mathbf{C}^{i}(i=\{1$, $2\}$ ), i.e., mode $i$, respectively. The phenomenological parameter $\theta \in[-1,1]$ quantifies the strength of the mode coupling (Edwards et al. 1996). Equation 7 represents the microstructural dynamics of mode $1, \mathbf{C}^{1}$. The time derivatives on the left side, together with the first three terms on the right side of Eqs. 7 and 8, represent the upperconvected derivatives of the contravariant second-rank tensors, $\mathbf{C}^{1}$ and $\mathbf{C}^{2}$ (Oldroyd 1950). The last three terms on the right side of Eqs. 7 and 8 describe the relaxation behavior of the internal microstructure (Edwards et al. 1996). The fourth and fifth terms are the relaxation terms of the Upper-Convected Maxwell Model and the sixth term accounts for the coupling between the microstructural variables. When $\theta=0$, a multiple uncoupled mode version of the Upper-Convected Maxwell Model is manifested.

The extra stress tensor for the TCMM Model is (Beris and Edwards 1994; Edwards et al. 1996)

$\boldsymbol{\sigma}=\sum_{i=1}^{2} G_{i}\left(\frac{K_{i}}{k_{\mathrm{B}} T} \mathbf{C}^{i}-\mathbf{1}\right)$

where $G_{i}=n_{i} k_{\mathrm{B}} T$ is the elastic modulus belonging to microstructural variable $\mathbf{C}^{i}(i=\{1,2\})$. It arises due to the fact that the microstructural components of the fluid are modeled as Hookean springs. Equations 1, 2, 7, 8 and 9 are thermodynamically consistent time evolution equations for the TCMM fluid. In the present article, we solve the Pompon, the Pom-Pom, and the TCMM models for a superposition of drag (Couette) flow with plane pressuredriven (Poiseuille) flow in Cartesian and cylindrical coordinates. In Cartesian coordinates, we examine flows between parallel plates, whereas in cylindrical coordinates, we study tube and Taylor-Couette flows. Therefore, the dynamical equations will be solved numerically to match prescribed boundary conditions on the walls. In this paper, we want to assume no-slip boundary conditions to be imposed on the velocity field at the boundaries. Mathematically, this is a TPBV problem because the physical fields depend on one spatial coordinate and have to match prescribed boundary conditions at the limits of the integration interval. The methodology to solve the boundary value problem is straightforward and computer algorithms are available (Press et al. 1992, cf., e.g.). However, it is not immediately obvious how to manipulate the three sets of continuum equations (Eqs. 1, 2, 3, 4 and 5 ) to obtain a computationally tractable formulation of the boundary value problem. The method is explained in the subsequent sections for the different flow geometries.

\section{Channel and tube flows}

Boundary conditions and non-dimensional system equations

In this section, the three viscoelastic fluid models (Pompon, Pom-Pom, and TCMM) will be solved for mixed Poiseuille and Couette flow between two horizontal planes located at $x_{2}=0$ and $x_{2}=H$ assuming steady laminar flow in the $x_{1}$ direction. Under these assumptions, the velocity vector is of the form $\mathbf{v}=v_{1}\left(x_{2}\right) \mathbf{e}_{1}$ and obeys the boundary conditions

$v_{1}\left(x_{2}=0\right)=0$

$v_{1}\left(x_{2}=H\right)=V$

i.e., the upper plane moves at a velocity $V$ relative to the lower one. The backbone orientation tensor, backbone stretch, conformation tensors, and extra stress tensors are functions of the transversal coordinate, $x_{2}$, and the density, $\rho$, is constant as $\boldsymbol{\nabla} \cdot \mathbf{v}=0$. For pure Poiseuille flow, i.e., $V=0$, the flow problem is symmetric with respect to the mid-plane, $x_{2}=$ $H / 2$, and the boundary conditions are

$v_{1}^{\prime}\left(x_{2}=H / 2\right)=0$,

$v_{1}\left(x_{2}=H\right)=0$

where "'" denotes differentiation with respect to the transversal direction, $x_{2}$. In the following paragraphs, we give the dimensionless form of the system equations for the three viscoelastic fluid models.

The Pompon and Pom-Pom equations are rendered dimensionless with $\widetilde{\mathbf{v}}=\sqrt{\lambda_{1} \lambda_{2}} \mathbf{v} / H, \widetilde{p}=p /(C g), \widetilde{\boldsymbol{\sigma}}=\boldsymbol{\sigma} /(C g)$, and $\widetilde{\mathrm{r}}=\mathrm{r} / H, \widetilde{\boldsymbol{\nabla}}=H \boldsymbol{\nabla}$. For tube flow, the channel height, $H$, is to be replaced with the tube radius, $R$. In what follows, we drop the " $\sim$ " denoting dimensionless quantities. Then, Eqs. 1, 2, 3, 4 and 5 reduce to the set of Pompon equations

$\boldsymbol{\nabla} \cdot\left(\lambda^{2} \mathbf{S}\right)-\boldsymbol{\nabla} p=0$

$\mathbf{S} \cdot \mathbf{L}^{\mathrm{T}}+\mathbf{L} \cdot \mathbf{S}-\frac{1}{\left(1+a_{1}\right) \widetilde{\lambda}_{1}}\left\{3 \mathbf{S} \cdot \mathbf{S}+\left(a_{1}-1\right) \mathbf{S}-\frac{a_{1}}{3} \mathbf{1}\right\}$
$=0$,

$\lambda \mathbf{L}: \mathbf{S}-\frac{1}{\widetilde{\lambda}_{2}}(\lambda-1) e^{\nu^{*}(\lambda-1)}=0$, 
where $\tilde{\lambda}_{1}=\sqrt{\lambda_{\mathrm{b}} / \lambda_{\mathrm{s}}}, \tilde{\lambda}_{2}=\sqrt{\lambda_{\mathrm{s}} / \lambda_{\mathrm{b}}}$. Equation 14 is obtained after inserting the stress constitutive assumption (Eq. 5) into the Cauchy momentum balance (Eq. 2). The equilibrium $(\mathbf{L}=0)$ solution of the above Eqs. 15 and 16 is $\mathbf{S}=1 / 3, \lambda=1$. With Eq. 5, this implies $\boldsymbol{\sigma}=1 / 3$. For the Pom-Pom Model, Eq. 15 is replaced with

$$
\mathbf{A} \cdot \mathbf{L}^{\mathrm{T}}+\mathbf{L} \cdot \mathbf{A}-\frac{1}{\widetilde{\lambda}_{1}}(\mathbf{A}-\mathbf{1})=0
$$

Note that the Pompon equations can be expressed in terms of the backbone orientation tensor, $\mathbf{S}$, whereas the Pom-Pom equations have to be expressed in terms of the second-rank tensor, $\mathbf{A}$, which has no strict physical meaning.

The TCMM equations are rendered dimensionless with $\quad \widetilde{\mathbf{v}}=\sqrt{\lambda_{1} \lambda_{2}} \mathbf{v} / H, \widetilde{\mathbf{C}}^{i}=K_{i} \mathbf{C}^{i} / k_{B} T \quad(i \in\{1,2\})$, $\widetilde{p}=p / \sqrt{G_{1} G_{2}}, \widetilde{\boldsymbol{\sigma}}=\boldsymbol{\sigma} / \sqrt{G_{1} G_{2}}$, and $\widetilde{\mathrm{r}}=\mathrm{r} / H, \widetilde{\boldsymbol{\nabla}}=H \nabla$.

Again, we drop the “ $\sim$ " denoting dimensionless quantities. Then, Eqs. 1, 2, 7, 8 and 9 reduce to the set of differential equations

$$
\begin{aligned}
& \sum_{i=1}^{2} \widetilde{G}_{i} \boldsymbol{\nabla} \cdot \mathbf{C}^{i}-\boldsymbol{\nabla} p=0 \\
& \mathbf{C}^{i} \cdot \mathbf{L}^{\mathrm{T}}+\mathbf{L} \cdot \mathbf{C}^{i}-\frac{1}{\widetilde{\lambda}_{i}} \mathbf{C}^{i}+\frac{1}{\widetilde{\lambda}_{i}} \mathbf{1} \\
& \quad-\frac{\theta}{2 \widetilde{G}_{i}}\left(\mathbf{C}^{1} \cdot \mathbf{C}^{2}+\mathbf{C}^{2} \cdot \mathbf{C}^{1}-2 \mathbf{C}^{i}\right)=0,
\end{aligned}
$$

where $\widetilde{\lambda}_{1}=\sqrt{\lambda_{1} / \lambda_{2}}, \widetilde{G}_{1}=\sqrt{G_{1} / G_{2}}$. Analogous definitions apply for $\widetilde{\lambda}_{2}$ and $\widetilde{G}_{2}$. Equation 18 is obtained by inserting the stress constitutive Eq. 9 into the Cauchy momentum balance (Eq. 2).

\section{Analytical solution of the 2MUCM model}

In this section, for $\theta=0$, the TCMM Eqs. 18 and 19 reduce to the 2MUCM Model, which is solved here analytically for laminar channel flow. The solution of the $v_{1}$-component of the momentum balance Eq. 18 reads

$$
v_{1}=-\frac{1}{2 \widetilde{\eta}} \frac{\partial p}{\partial x_{1}} x_{2}\left(H-x_{2}\right)+\frac{V x_{2}}{H},
$$

where $\widetilde{\eta}=\widetilde{G}_{1} \widetilde{\lambda}_{1}+\widetilde{G}_{2} \widetilde{\lambda}_{2}$ and $\partial p / \partial x_{1}$ is the constant pressure drop in the direction of flow. For the conformation tensor Eq. 19, we arrive at the solutions

$C_{11}^{i}=2 \widetilde{\lambda}_{j}^{2}\left[-\frac{1}{2 \widetilde{\eta}} \frac{\partial p}{\partial x_{1}}\left(H-2 x_{2}\right)+\frac{V}{H}\right]^{2}+1$,

$C_{12}^{i}=-\frac{\widetilde{\lambda}_{i}}{2 \widetilde{\eta}} \frac{\partial p}{\partial x_{1}}\left(H-2 x_{2}\right)+\frac{\widetilde{\lambda}_{i} V}{H}$,

$C_{j j}^{i}=1, j=\{2,3\}$,

and all other $C_{j k}^{i}=0$ for $i=\{2,3\}$. Note that, for the 2MUCM Model, we have $\partial p / \partial x_{2}$ from the $v_{2}$-component of Eq. 18 together with Eq. 23. The extra stress tensor can be calculated from the above Eqs. 21, 22 and 23 using the constitutive relationship of Eq. 9. The volumetric flow rate, $Q$ (volume/length), through a cross section of the channel perpendicular to the $x_{1}$-axis is

$Q=\int_{0}^{W} \mathrm{~d} x_{3} \int_{0}^{H} v_{1} \mathrm{~d} x_{2}=-\frac{1}{12 \widetilde{\eta}} \frac{\partial p}{\partial x_{1}} H^{3} W+\frac{V}{2} H W$,

where $W$ is a unit length in the neutral direction, $\mathbf{e}_{3}$, and $v_{1}$ is given by Eq. 20. From Eq. 24, we recognize that a positive drag flow, $V$, gives a positive contribution and a positive pressure drop, $\partial p / \partial x_{1}$, gives a negative contribution to the total volumetric flow rate. For a given drag velocity, $\mathbf{V}=V \mathbf{e}_{1}$, the volumetric flow rate (Eq. 24) vanishes for

$$
\left(\frac{\partial p}{\partial x_{1}}\right)^{\dagger}=\frac{6 \widetilde{\eta}}{H^{2}} V
$$

To describe the axial flow of a 2MUCM fluid through a circular tube of radius $R$, Eqs. 18 and 19 have to be solved in cylindrical coordinates $(r, \varphi, z)$, where $r$ is the distance from the cylinder axis, $\varphi$ is the azimuthal coordinate, and $z$ is the axial coordinate along the cylinder axis. The solution of the continuum Eqs. 18 and 19 is analogous to the symmetric channel flow problem, which is given in the "Appendix". From the solution of the tube flow problem, Eqs. 72 and 73, we obtain

$$
Q=\int_{0}^{2 \pi} d \varphi \int_{0}^{R} v_{z} r \mathrm{~d} r=-\frac{\pi}{8 \widetilde{\eta}} \frac{\partial p}{\partial z} R^{4}
$$


which corresponds to Hagen-Poiseuille's law. The wall extra stresses resulting from the non-equilibrium conformations, Eq. 73 in the "Appendix", lead to a die swell which can be estimated from the wall viscometric properties (Bird et al. 1987):

$\frac{D^{\prime}}{D}=\frac{1}{10}+\left[\mathbf{1}+\frac{1}{2}\left(\frac{\sigma_{z z, \mathrm{w}}-\sigma_{r r, \mathrm{w}}}{2 \sigma_{r z, \mathrm{w}}}\right)^{2}\right]^{\frac{1}{6}}$.

$\mathcal{A} D=2 R$ is the diameter of the tube, $D^{\prime}$ is the diameter of the swollen fluid, and the subscript " $w$ " refers to the wall viscometric properties. For the $2 \mathrm{MUCM}$ model, we get from Eq. 27 with Eq. 73

$$
\frac{D^{\prime}}{D}=\frac{1}{10}+\left[1+\frac{1}{2}\left(\frac{\widetilde{\eta}_{2}}{2 \widetilde{\eta}^{2}} \frac{\partial p}{\partial z} R\right)^{2}\right]^{\frac{1}{6}},
$$

where $\widetilde{\eta}_{2}=\widetilde{G}_{1} \widetilde{\lambda}_{1}^{2}+\widetilde{G}_{2} \widetilde{\lambda}_{2}^{2}$. These solutions can be compared with the numerical results obtained with the TCMM Model for $\theta=0$ to validate the proposed numerical methodology. In all cases examined in this study, such a validation was perfect.

In the next section, we explain how the three viscoelastic fluid models have been reformulated in terms of TPBV problems to solve for laminar channel and tube flow. For the channel geometry, the TPBV problems are solved for mixed Couette and Poiseuille flow. The tube flow problems are solved for pure Poiseuille flow.

\section{Numerical method}

In this section, we explain how the three models have been discretized and solved numerically for superimposed Couette and Poiseuille flow.We explain the methodology separately for the three models considering both channel and tube flows. First, we consider the channel flow problem for the three viscoelastic fluid models.

For a superposition of unidirectional and laminar Poiseuille flow with Couette flow, the three sets of PDEs (Eqs. 14, 15, 16, 17, 18 and 19) reduce to sets of ODEs

$\mathcal{A} \cdot \boldsymbol{\xi}^{\prime}=\mathbf{b}$,

which have to be solved for the boundary conditions (Eqs. 10 and 11 or Eqs. 12 and 13) depending on whether the channel flow profiles are non-symmetric or symmetric with respect to the mid-plane. In the above linear equation, $\boldsymbol{\xi}$ is a $n$-tuple of non-trivial physical fields, $\mathcal{A}$ is a $n \times n$ coefficient matrix, b is another $n$-tuple called the "inhomogeneity," and "'" denotes differentiation with respect to the $x_{2}$ coordinate. The coefficient matrix, $\mathcal{A}$, and the inhomogeneity, $\mathbf{b}$, have to be obtained from the continuum Eqs. 14, 15, 16, 17, 18 and 19. For laminar channel flow, we have

$\boldsymbol{\xi}=\left(v_{1}, v_{1}^{\prime}, p, X_{11}, X_{12}, X_{22}, \lambda\right)^{\mathrm{T}}$,

$\boldsymbol{\xi}=\left(v_{1}, v_{1}^{\prime}, p, C_{11}^{1}, C_{12}^{1}, C_{22}^{1}, C_{11}^{2}, C_{12}^{2}, C_{22}^{2}\right)^{\mathrm{T}}$,

where $v_{1}^{\prime}=\partial v_{1} / \partial x_{2}$ is the velocity gradient. Equation 30 is for the Pompon Model $\left(X_{i j} \equiv S_{i j}\right)$ or the Pom-Pom Model $\left(X_{i j} \equiv A_{i j}\right)$, and Eq. 31 is for the TCMM Model. The coefficient matrix, $\mathcal{A}$, and the inhomogeneity, $\mathbf{b}$, are identified from the system Eqs. 14, 15, 16, 17, 18 and 19.

For the TCMM Model, the coefficient matrix, $\mathcal{A}$, and the inhomogeneity, $\mathbf{b}$, are obtained in the following way. The first two rows of $(\mathcal{A}, \mathbf{b})$ corresponding to $v_{1}$ and $v_{1}^{\prime}$ are the $v_{1}$ component of Eq. 18 and the $C_{12}^{i}$-component of Eq. 19 (either for $i=1$ or 2), respectively. The third row of $(\mathcal{A}, \mathbf{b})$ corresponds to the $v_{2}$-component of the momentum balance Eq. 18. The remaining six rows of $(\mathcal{A}, \mathbf{b})$ corresponding to the non-trivial components of the conformation tensor are identified by taking the derivative of the appropriate component of the conformation tensor Eq. 19 with respect to $x_{2}$.

The TPBV problems for the three viscoelastic fluid models are solved computationally using a shooting algorithm. The algorithm uses a fourth-order Runge-Kutta scheme with adaptive step size to integrate the set of ODEs and a globally convergent Newton algorithm to match the imposed boundary conditions at the upper channel wall, $x_{2}=H$. First, in our computer algorithm the linear system (Eq. 29) is solved using an LU decomposition of the coefficient matrix, $\mathcal{A}$. The set of ODEs $\boldsymbol{\xi}^{\prime}=\mathcal{A}^{-1} \cdot \mathbf{b}$ is then integrated with the Runge-Kutta algorithm shooting from the lower plane, $x_{2}=0$, to the upper plane, $x_{2}=H$. Every Runge-Kutta shoot starts with the same initial condition (Eq. 10) on the velocity field and a different wall shear rate, $v_{1}^{\prime}\left(x_{2}=0\right)$, being the independent variable in the Newton subroutine of the shooting algorithm. The Runge-Kutta integrations are repeated until the Newton algorithm has converged, i.e., the boundary condition on the upper plane, (Eq. 11), is satisfied. Note that, before every Runge-Kutta shot, the homogeneous shear flow problem related to Eq. 19 has to be solved for $v_{1}^{\prime}\left(x_{2}=0\right)$ because the structural variables on the lower plane are non-trivial functions of the wall shear rate. This is necessary because the symmetry of the flow with respect to $x_{2}=H / 2$ is broken in the case of mixed Poiseuille and Couette flow. 
For tube flow, the three sets of model equations are reformulated in terms of TPBV problems (Eq. 29) for the boundary conditions Eqs. 69 and 70 in the "Appendix". The $n$-tuples of unknown profiles now are

$\boldsymbol{\xi}=\left(p, v_{z}, v_{1}^{\prime}, X_{r r}, X_{r z}, X_{z z}, \lambda\right)^{\mathrm{T}}$,

$\boldsymbol{\xi}=\left(p, v_{z}, v_{1}^{\prime}, C_{r r}^{1}, C_{r z}^{1}, C_{z z}^{1}, C_{r r}^{2}, C_{r z}^{2}, C_{z z}^{2}\right)^{\mathrm{T}}$,

where Eq. 32 is for the Pompon Model $\left(X_{i j} \equiv S_{i j}\right)$ or the PomPom Model $\left(X_{i j} \equiv A_{i j}\right)$ and Eq. 33 is for the TCMM Model. The "'" denotes differentiation with respect to the radial coordinate. The coefficient matrices, $\mathcal{A}$, and the inhomogeneities, $\mathbf{b}$, are obtained in an analogous way as for the laminar channel flow profiles. Note that, for the symmetric tube flow problem, it is not necessary to solve numerically the steady-state flow problem before every Runge-Kutta shoot as the velocity gradient vanishes on the tube axis, i.e., $\mathbf{S}=\mathbf{1} / 3, \lambda$ $=1 ; \mathbf{C}^{i}=1(i=\{1,2\})$ for $r=0$ in the three viscoelastic fluid models. In the computer code, the velocity on the tube axis is the independent variable of the Newton subroutine in the shooting algorithm. It is varied until the boundary condition (Eq. 70) is matched.

The accuracy of our solution technique has been verified for the TCMM Model in the limit where this model reduces to the 2MUCM Model $(\theta=0)$. As already mentioned, the 2MUCM Model has analytical solutions in the three geometries considered in this article. For a sufficiently refined mesh, the computational code reproduced the analytical results, within machine precision. The RungeKutta integrator used in the shooting algorithm had an adaptive step size.

Sample calculations for channel and tube flow are presented and discussed in the following section. This is done to illustrate the efficiency of the numerical scheme, to discuss qualitatively the model behavior, and to compare solutions of the three models. Furthermore, we will address numerical problems which have been encountered in the numerical solution of the Pom-Pom Model.

\section{Sample calculations}

\section{The Pom-Pom and Pompon models}

The boundary value problems for laminar channel and tube flow of the Pompon Model and the Pom-Pom Model have been solved computationally for $\lambda_{\mathrm{b}} / \lambda_{\mathrm{s}}=3$ and various values for the number of arms, $q$. For the sample calculations of the Pompon Model, we chose $a_{1}=1$. The results of the TPBV calculations are reported in Figs. 1, 2, 3, 4 and 5 .
Figure 1 displays the velocity, stress tensor, and backbone stretch profiles for laminar channel flow of a Pompon fluid at five pressure drops. As the relative velocity of the two planes is zero $(V=0)$, we solved the TPBV problem for the boundary conditions Eqs. 12 and 13 exploiting the symmetry of the fields. In Fig. 3, we will present velocity and shear rate profiles for mixed Couette and Poiseuille flow between horizontal planes. The velocity field (Fig. 1a, $v \equiv v_{1}$ ) and the normal stresses (Fig. 1d,e) are symmetric with respect to the channel mid-plane; the shear rate (Fig. $1 \mathrm{~b}, \dot{\gamma}=v_{1}^{\prime}$ ) and the shear stress (Fig. 1c) are anti-symmetric. On the mid-plane of the channel, we have $\sigma=1 / 3$ and $\lambda=1$. Note that the transversal normal stresses (Fig. 1e) are positive and show a non-monotonic behavior, which is qualitatively different from the results found for the TCMM Model (cf. Fig. 6). The backbone stretch (Fig. 1f) increases monotonically proceeding from the mid-plane to the upper wall, and it is symmetric with respect to the channel mid-plane. The normal stress in the vorticity direction is $\sigma_{33}=1 / 3 \lambda^{2}$ as $S_{33}$ is at its equilibrium value for laminar channel flow. From Fig. 1, we note that the shear rate, the components of the extra stress tensor, and the backbone stretch assume their maximum values on the channel wall. The wall viscometric properties are, thus, non-trivial functions of the wall shear rate. These properties are reported in Fig. 2 and will be discussed in the next paragraph. The influence of the number of arms, $q$, on the macroscopic flow behavior of the Pompon fluid is discussed in Fig. 4.

In Fig. 2, the nonlinear viscometric functions of the Pompon Model are plotted as functions of the negative wall shear rate on the upper wall of the channel. The shear stress is a monotonically increasing function of the shear rate, and we observe a typical viscoelastic response with a shear-thinning behavior of the viscosity and the first normal stress coefficient. The second normal stress coefficient is negative, and it varies between $\Psi_{2 \mathrm{w}} / \Psi_{1 \mathrm{w}}=-1 /\left(2\left(1+\mathrm{a}_{1}\right)\right)$ for small shear rates and zero for large shear rates. Note that the results reported in Fig. 2 are identical to the nonlinear viscometric functions as obtained from solution of the steady-state homogeneous shear flow problem. The reason for this is that the backbone orientation tensor and the backbone stretch on the channel wall must satisfy Eqs. 3 and 4 for the wall shear rate, $\dot{\gamma}_{\mathrm{w}}$, which is obtained from solution of the TPBV problem.

Figure 3 shows the velocity field for Poiseuille flow superimposed onto Couette flow for $q=3$ and various pressure drops, $\nabla_{1} p$, denoted with each curve. In Fig. 3a, we show the velocity $\left(v \equiv v_{1}\right)$ as a function of the channel height, and in Fig. 3b, we display the shear rate $\left(\dot{\gamma} \equiv v_{1}^{\prime}\right)$. The vertical line $v=0$ in Fig. $3 \mathrm{a}$ is to indicate where the velocity changes its sign. The relative velocity of the planes is $V=0.5$, as can be identified from Fig. 3a. As the flow problem is not symmetric, the TPBV problem has been solved with the boundary conditions (Eqs. 10 and 11) so that the steady-state shear flow problem is solved on the lower 
Fig. 1 Laminar channel flow profiles for the Pompon Model at five pressure drops: $\nabla_{1} p=-2$ (solid lines), -4 (dotted lines), -6 (dashed lines), -8 (longdashed lines), and -10 (dotdashed lines). Model parameters are $\lambda_{\mathrm{b}} / \lambda_{\mathrm{s}}=3, a_{1}=1, q=3$. a Velocity profile, $\mathbf{b}$ shear rate, c shear stress, d normal stress in flow direction, e normal stress in transversal direction, and f backbone stretch
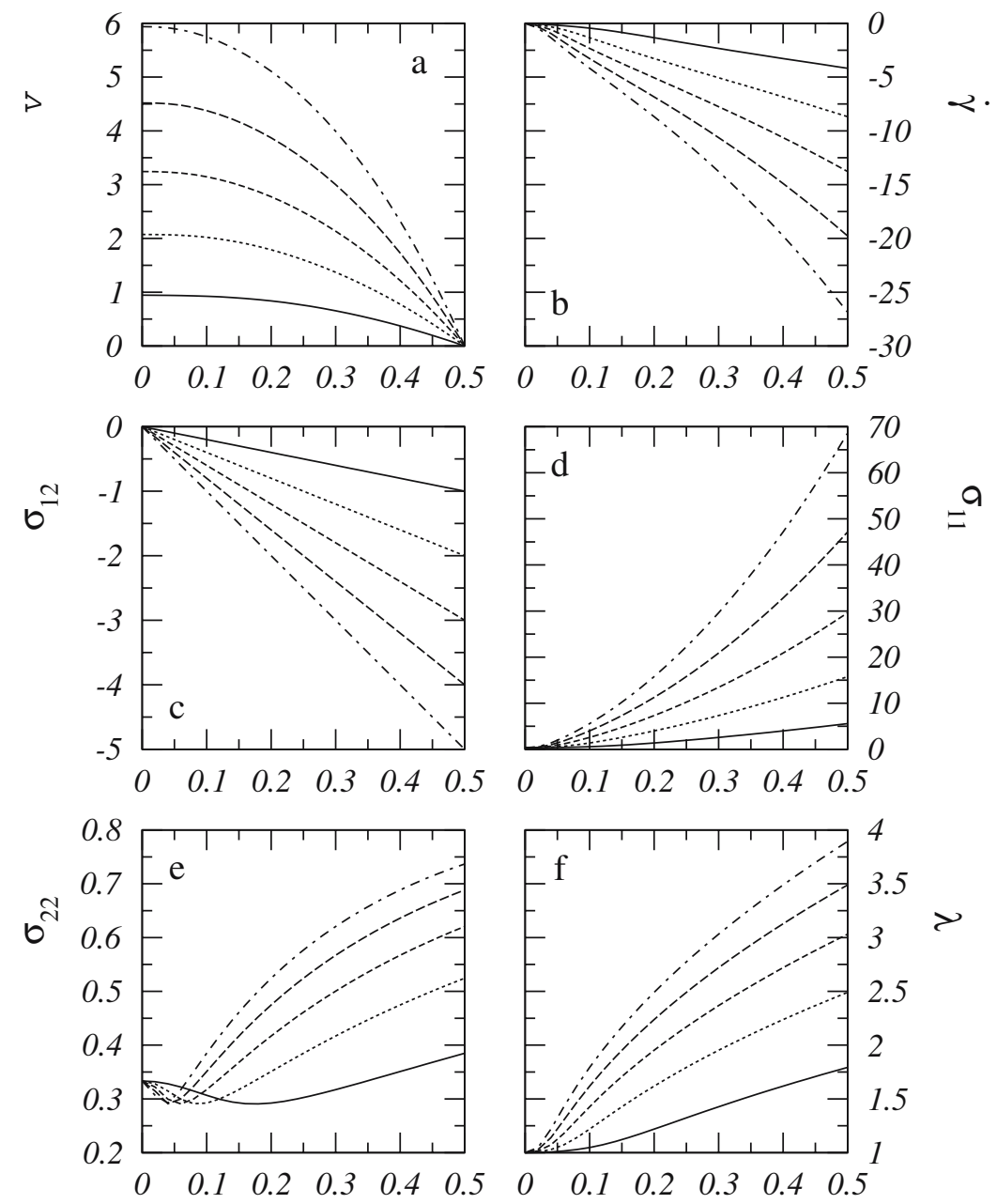

distance from mid-plane, $x_{2}$ plate before every Runge-Kutta shoot to yield a correct starting $n$-tuple, $\xi$, for the Runge-Kutta integration. In Fig. 3a, we observe that the positive drag flow, $V=0.5$, gives a positive volumetric flow rate and the positive pressure drop, $\nabla_{1} p$, gives a negative volumetric flow rate. Consequently, for every velocity of the upper plane, one can identify a critical pressure drop, $\left(\nabla_{1} p\right)^{\dagger}$, which leads to a vanishing total volumetric flow rate (cf. the discussion for the 2MUCM Model and the related Eq. 27 above).

Figure 4 displays the maximum fluid velocity (Fig. 4a), the wall shear rate (Fig. 4b), and the volumetric flow rate (Fig. 4c) for tube flow of a Pompon fluid. The volumetric flow rate has been calculated numerically using a ten-point Gauss-Legendre integration (Press et al. 1992) of the velocity profile. We have considered a stationary (nonrotating and non-translating) tube wall, and we show sample calculations for three Pompon chains with different numbers of arms. We observe that the behavior of the volumetric flow rate correlates with the maximum fluid velocity on the tube axis and the wall shear rate.
Furthermore, the maximum fluid velocity, wall shear rate, and volumetric flow rate decrease as the number of arms increases. These three quantities increase monotonically with the applied pressure drop.

In what follows, we discuss the steady-state shear flow properties of the Pom-Pom Model for one set of model parameters, $\lambda_{\mathrm{b}} / \lambda_{\mathrm{s}}$ and $q$. We solve this model for the steady-state homogeneous shear flow problem and the laminar channel flow problem. The wall viscometric material properties obtained from the two numerical schemes will be discussed as above for the Pompon Model (cf. Fig. 2).

Figure 5 shows the viscometric material properties for laminar channel flow of a Pom-Pom fluid $\left(\lambda_{\mathrm{b}} / \lambda_{\mathrm{s}}=3\right.$ and $q=3$ ) as a function of the negative wall shear rate on the upper wall of the channel. Note that the wall shear stress according to this model is a non-monotonic function of the wall shear rate, which is qualitatively different from the prediction of the Pompon Model and the TCMM Model in Figs. 2 and 7. In fact, this model displays the completely 


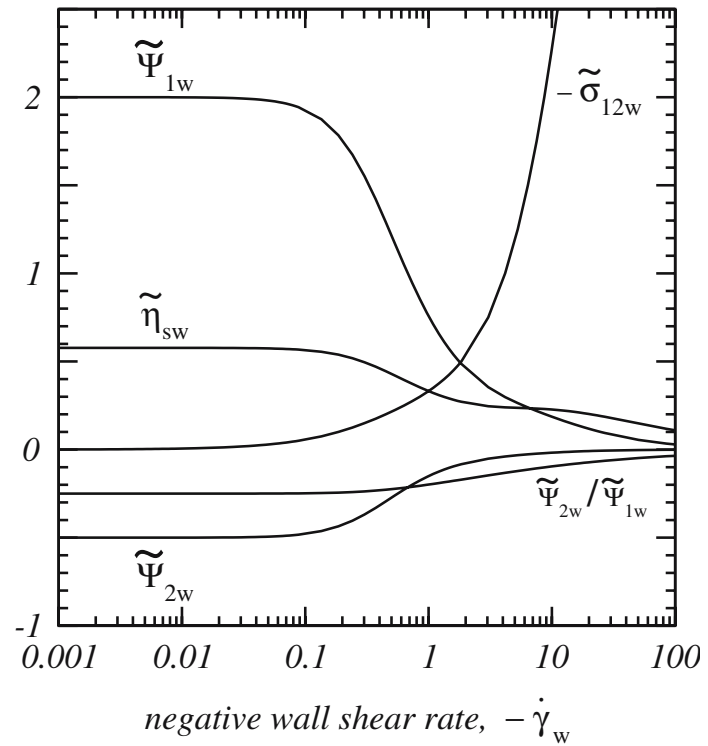

Fig. 2 Non-linear viscometric functions according to the Pompon Model as found in laminar channel flow. Model parameters are the same as in Fig. 1
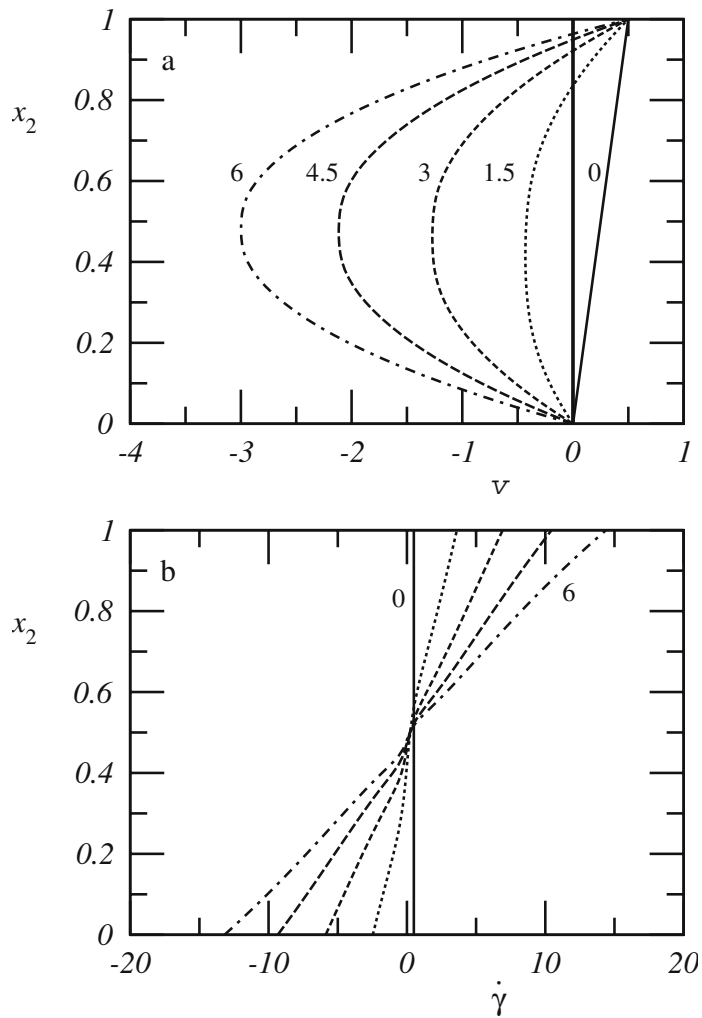

Fig. 3 Velocity (a) and shear rate (b) profiles for superimposed Poiseuille and drag flow of a Pompon fluid. The velocity of the upper plane is $V=0.5$ and the positive pressure gradient is denoted with each curve. Model parameters are the same as in Fig. 1



Fig. 4 Maximum fluid velocity (a), wall shear rate (b), and volumetric flow rate (c) for laminar tube flow of a Pompon fluid at three values of the arm parameter: $q=3$ (solid lines), 30 (dotted lines), and 300 (dashed lines). Other model parameters are the same as in Fig. 1

absurd result that the shear stress in homogeneous steadyshear flow goes to zero with increasing shear rate. (For homogeneous flow, replace $\dot{\gamma}_{\mathrm{w}}$ with $-\dot{\gamma}$ in the figure). This seems to be an artifact of the model and not a result of any unusual flow behavior, such as shear band formation (which would have been evident in the figures discussed previously). Given the definition of $\mathbf{S}$ ( $=\mathbf{A} / \operatorname{tr} \mathbf{A}$ ) which appears in the expression for $\boldsymbol{\sigma}$, it is evident that $A_{12}$ becomes much smaller than trA as the molecular conformations extend with increasing shear rate.

The second normal stress coefficient is zero for the PomPom Model. The solid lines in Fig. 5 have been obtained from the solution of the TPBV problem for the Pom-Pom fluid using the shooting algorithm. The dotted lines, which superimpose onto the solid lines in the low shear rate regime, have been obtained from solution of the steady-state homogeneous shear flow problem. Note that the solution of the steady-state homogeneous shear flow problem, which has been obtained with a Newton Raphson algorithm (Press et al. 1992), can be calculated over the entire range of shear rates shown in Fig. 5. However, the solid lines, which have been 


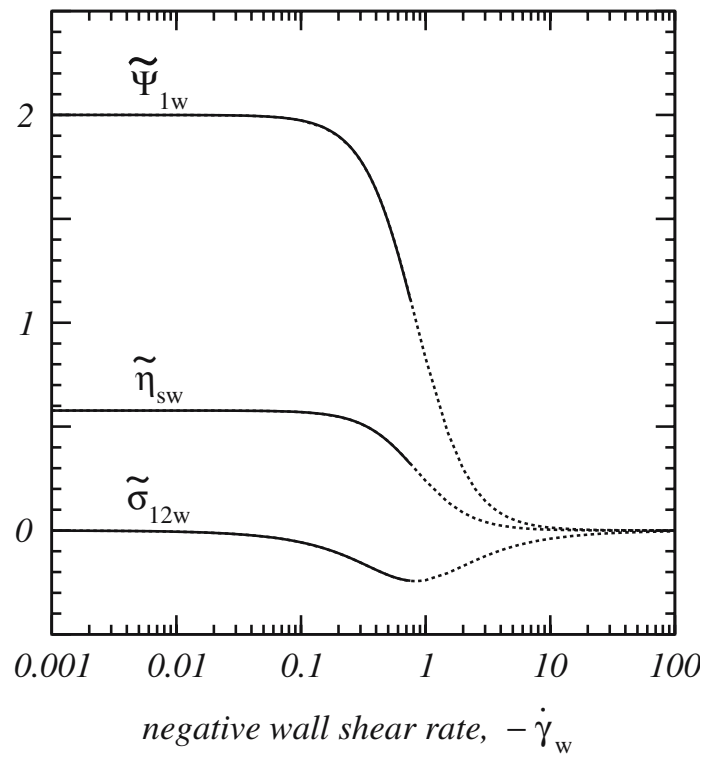

Fig. 5 Non-linear viscometric functions according to the Pom-Pom Model as found from solution of the steady-state homogeneous flow problem (dotted lines) and from solution of the TPBV problem (solid lines). Model parameters are $\lambda_{\mathrm{b}} / \lambda_{\mathrm{s}}=3$ and $q=3$. The shear stress vs shear rate dependence is non-monotonic

obtained from the shooting algorithm, can be calculated only up to the shear rate $\dot{\gamma}^{*}$, where the wall shear rate has a minimum $\partial \sigma_{12} / \partial \dot{\gamma}_{\mathrm{w}} \dot{\gamma}^{*}=0$. The reason for this breakdown of the shooting algorithm in the vicinity of the wall shear rate, $\dot{\gamma}^{*}$, lies in the first equation of the linear system (Eq. 29), which corresponds to the $v_{1}$-component of the Cauchy momentum balance (Eq. 2),

$\frac{\partial \sigma_{12}}{\partial x_{2}}=\frac{\partial p}{\partial x_{1}}$.

Using the stress constitutive relationship (Eq. 5), the left side of Eq. 34 can be identified with the left side of the linear Eq. 29, $\sum_{i=1}^{7} \mathcal{A}_{1 i} \xi_{i}, b_{7}=\partial p / \partial x_{1}$. However, the left side of Eq. 34 can be rewritten as

$\left.\frac{\partial \sigma_{12}}{\partial x_{2}}\right|_{\widetilde{x}_{2}}=\left.\left.\frac{\partial \sigma_{12}}{\partial v_{1}^{\prime}}\right|_{\widetilde{\gamma}} \frac{\partial v_{1}^{\prime}}{\partial x_{2}}\right|_{\widetilde{x}_{2}}$.

Now, for $v_{1}^{\prime}=\dot{\gamma}^{*}$, the left side of Eq. 34 is zero and the first row of the coefficient matrix A in Eq. 29 vanishes. Consequently, the minimum of the shear stress implies that the rank of the coefficient matrix, A, in Eq. 29 is reduced by one, which leads to the numerical instabilities of the shooting algorithm. In our computer code, the step size in the RungeKutta integrator of the shooting algorithm decreases towards zero and the integration of Eq. 29 stops as the shear rate approaches the value $\dot{\gamma}^{*}$. This occurs for pressure drops that lead to wall shear rates slightly below $\dot{\gamma}^{*}$, as seen in Fig. 5.

As a consequence of the non-monotonic shear stress vs shear rate predictions reported in Fig. 5 and the numerical instabilities of the shooting algorithm related to this model behavior, we do not consider the Pom-Pom Model further in the present article. Instead, we focus on the Pompon and the TCMM models as we are interested mainly in the model predictions for laminar flows through channels, tubes, and annular gaps. Presumably, the Pom-Pom Model gives similar flow profiles as the Pompon Model for shear rates below $\dot{\gamma}^{*}$. Furthermore, Chodankar et al. (2003) have shown that the Pompon Model provides a better qualitative match of the integral Pom-Pom Model in step-strain flows than the differential Pom-Pom Model. This implies that the Pompon Model might more accurately represent the physics contained within the more fine-grained integral version of the original Pom-Pom Model. The original Pom-Pom Model was extended by Verbeeten et al. (2001) to allow for the prediction of a non-zero second normal stress coefficient. As above, the Extended Pom-Pom Model can also be checked to see if it predicts a monotonic steady-state shear stress vs shear rate profile under homogeneous shear flow.

\section{The TCMM Model}

The boundary value problems for channel and tube flow of a TCMM fluid were also solved computationally, and sample calculations for $\lambda_{2} / \lambda_{1}=2, n_{2} / n_{1}=1$ and different values of the phenomenological coupling parameter, $\theta$, are reported in Figs. 6, 7 and 8. Figures 6 and 7 are for channel flow and Fig. 8 is for tube flow.

Figure 6 displays the velocity, extra stress tensor, and pressure profiles for laminar channel flow for five pressure drops. As the relative velocity of the two planes is zero $(V=0)$, we solve the TPBV problem for the boundary conditions (Eqs. 12 and 13) exploiting the symmetry of the physical fields. The velocity field $\left(v \equiv v_{1}\right)$ in Fig. 6a is symmetric with respect to the mid-plane and the shear rate profile in Fig. $6 \mathrm{~b}$ is antisymmetric $\left(\dot{\gamma} \equiv v_{1}^{\prime}\right)$. For the smallest pressure drop, the shear rate profile is almost linear, whereas for higher pressure drops, we observe a pronounced departure from linearity as the channel wall is approached. According to the momentum balance, the shear stress is linear and it vanishes on the mid-plane where the shear rate is zero (Fig. 6c). Figure 6d, e displays the normal stress profiles, which are even functions. We observe that the absolute value of the normal stresses acting on the channel walls $\left(\sigma_{22}\right)$ are two orders of magnitude smaller than the normal stresses acting in the direction of flow $\left(\sigma_{11}\right)$. A consequence of the appearance of the normal stresses, $\sigma_{22}$, is that one has to exert a normal force, $F / A=-\sigma_{22}$, on the planes to obtain a 
Fig. 6 Laminar channel flow profiles for a TCMM fluid $\left(\lambda_{2} / \lambda_{1}=2, n_{2} / n_{1}=1, \theta=0.1\right)$ at five pressure drops: $\nabla_{1} p=-2$ (solid lines), -4 (dotted lines), -6 (dashed lines), -8 (longdashed lines), and -10 (dotdashed lines). a Velocity profile, b shear rate, c shear stress, d normal stress in flow direction, $\mathbf{e}$ normal stress in transversal direction, $\mathbf{f}$ pressure
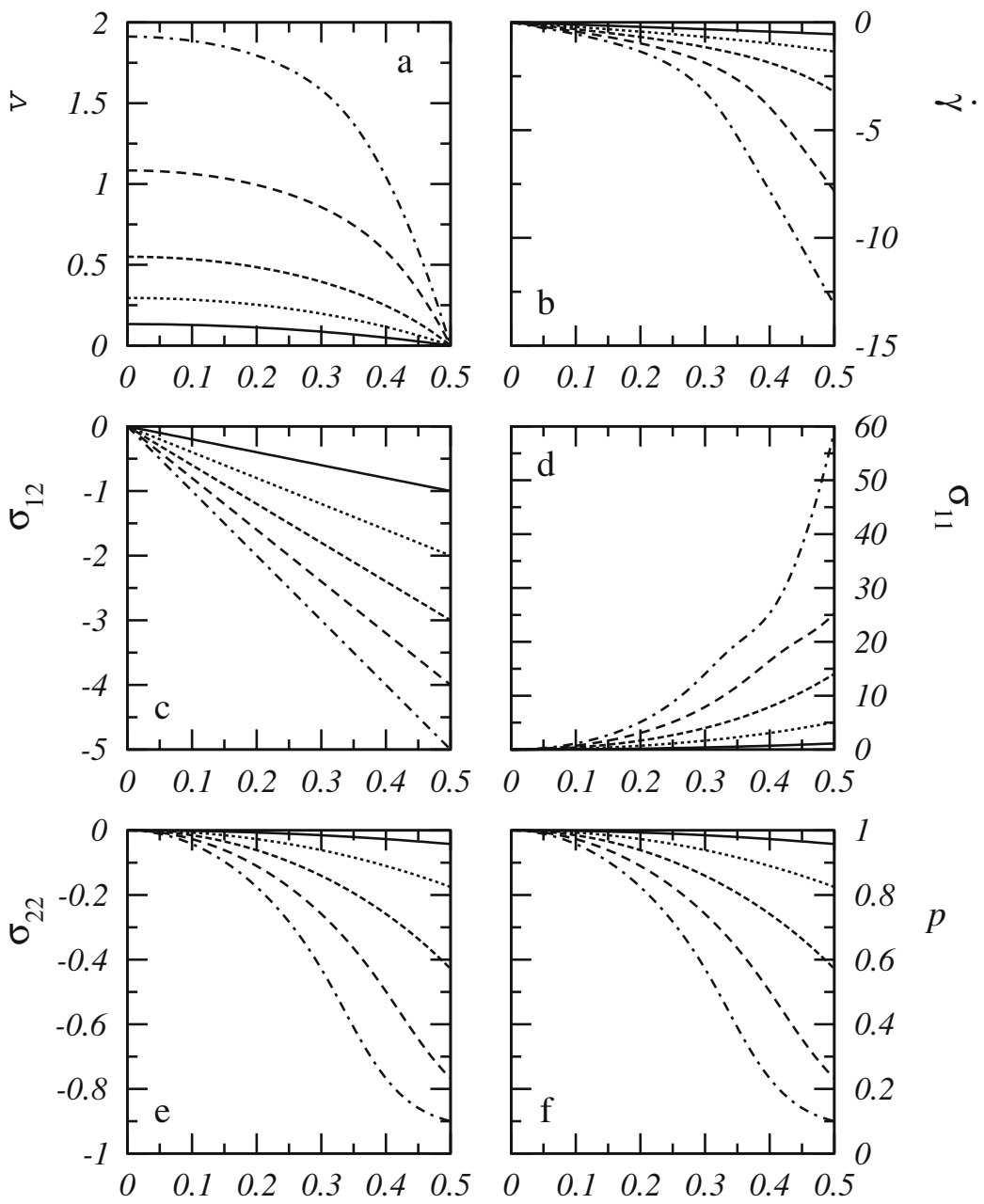

distance from mid-plane, $x_{2}$

steady-state channel flow. The normal stress in the vorticity direction vanishes, $\sigma_{33}=0$. The pressure profile is reported in Fig. 6f. Note that we are investigating here an incompressible fluid. Therefore, the pressure has no thermodynamic definition, but it ensures that the velocity field fulfills the incompressibility condition, $\boldsymbol{\nabla} \cdot \mathbf{v}=0$, along with the momentum balance equation. In Cartesian coordinates, we have $\nabla_{2} \sigma_{22}-\nabla_{2} p=0$, so that the pressure profile is identical to the transversal normal stress profile: $p=\sigma_{22}+p_{0}$, where $p_{0}$ is a constant.

Figure 7 displays the nonlinear viscoelastic properties of the TCMM fluid as a function of the negative wall shear rate on the upper wall. The model parameters are the same as before (in Fig. 6). We obtain a monotonic increase of the negative wall shear stress with the wall shear rate, and we observe a typical shear thinning behavior with a positive first normal stress coefficient and a negative second normal stress coefficient. Note that the nonlinear flow curves shown in Fig. 7 are obtained from the solution of the TPBV problem. We obtain identical flow curves as we obtained previously from the solution of the steady-state homogeneous flow problem (Edwards et al. 1996). The qualitative model behavior of the TCMM Model is identical to the Pompon Model (cf. Fig. 2): the shear stress is a monotonically increasing function of the shear rate, the first normal stress coefficient is positive, the second normal stress coefficient is negative, and the ratio of normal stress differences is less than unity. Contrarily, the Pom-Pom Model predicts a non-monotonic stress vs shear rate behavior and vanishing second normal stress difference.

In Fig. 8, we show the die swell, predicted according to Eq. 27, for the 2MUCM fluid and three non-trivial coupling parameter values of the TCMM fluid. We have studied pure Poiseuille flow, i.e., the tube wall is stationary (no translational or rotational motion of the tube is considered). For small pressure drops, the diameter of the swollen fluid increases slightly, whereas for large pressure drops, we get a strong increase of the relative die swell as a function of the pressure drop. The transition from the slight 


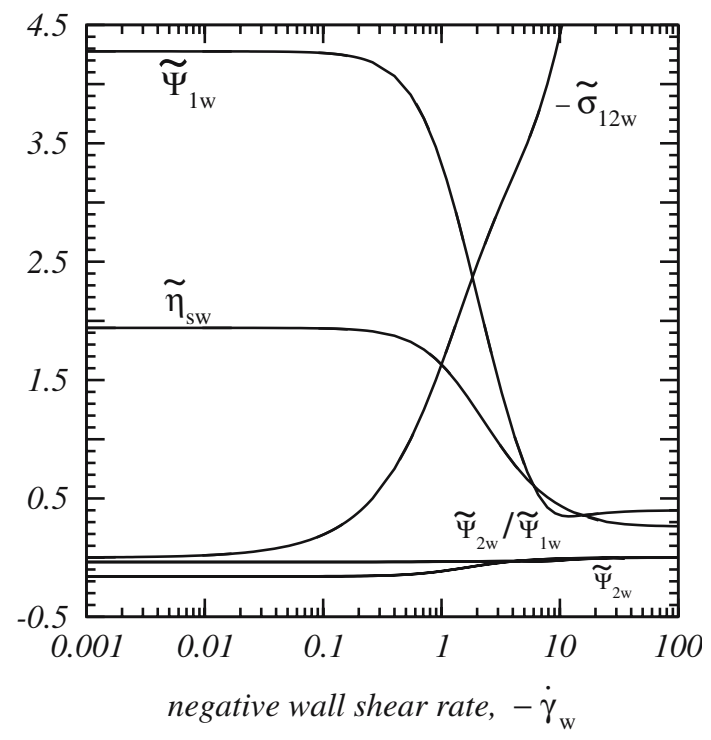

Fig. 7 Viscometric properties of a TCMM fluid evaluated at the channel wall as a function of wall shear rate. Model parameters are the same as in Fig. 6

to the strong extrudate swelling regime in Fig. 8 occurs at pressure drops slightly below $\nabla_{z} p=-10$. Note that the onset of significant die swell in Fig. 8 decreases as the nonlinear coupling parameter increases. This seems reasonable as the increasing coupling parameter value implies a broader separation of the characteristic times of the two relaxation modes (Edwards et al. 1996).

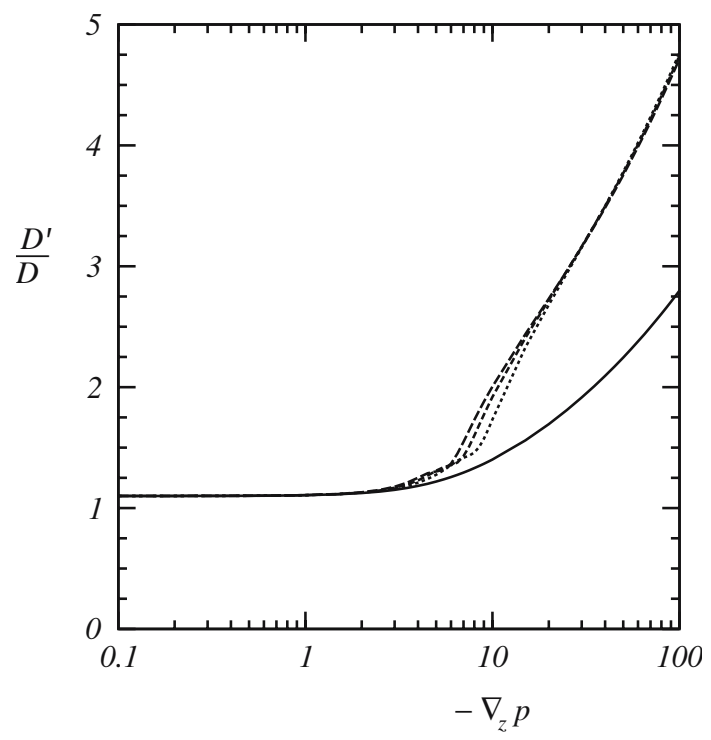

Fig. 8 Die swell predictions according to Eq. 27 for four coupling parameter values: $\theta=0$ (solid lines), 0.1 (dotted lines), 0.15 (dashed lines), and 0.2 (long-dashed lines). Other model parameters are the same as in Fig. 6
Comparison with experimental trends

There is not a great wealth of experimental data available concerning stress and velocity profiles in channel and tube flows. What does exist is in qualitative agreement with the model predictions described above, with a couple of exceptions. First, there is no experimental evidence to suggest that the non-monotonic steady-state wall shear stress profile of the Pom-Pom Model exhibited in Fig. 5 is physically reasonable. Indeed, the fact that this quantity vanishes for high flow rates seems particularly egregious. At first glance, this effect might potentially be ascribed to wall slip; however, the fact that the same quantitative behavior is observed for the steady-state shear stress vs shear rate in homogeneous shear flow clearly indicates that this behavior is an aphysical artifact of the differential Pom-Pom Model.

Second, the Pompon Model predicts a non-monotonic curve for $\sigma_{22}$ near the centerline of the channel. Of course, there is no experimental data available to suggest even a qualitative trend for this quantity, but we cannot imagine any potential mechanisms leading to this behavior. Perhaps a study of the underlying chain conformations would shed light on this issue. Nevertheless, this problem with the Pompon Model, assuming it is one, does not seem to affect the numerical stability of this model.

\section{Taylor-Couette flow}

Boundary conditions and dimensionless system equations

In this section, the Pompon and TCMM Model are solved for mixed Poiseuille and Couette flow through the annular gap between two concentric cylinders with inner radius $R_{\mathrm{i}}$ and outer radius $R_{\mathrm{o}}$. We explain how the coefficient matrix, $\mathcal{A}$, and the inhomogeneity, $\mathbf{b}$, change with respect to channel and tube flow. The ratio of cylinder radii is $k=R_{\mathrm{i}} / R_{\mathrm{o}}$, the annular gap is $\delta=R_{\mathrm{O}}-R_{\mathrm{i}}$, and the relative distance from the inner cylinder is $\bar{r}=\left(r-R_{i}\right) /\left(R_{\mathrm{O}}-R_{i}\right)$. Again, we adopt cylindrical coordinates $(r, \varphi, z)$ to describe the flow. The no-slip boundary conditions to be imposed on the velocity field are

$$
\begin{aligned}
& v_{\varphi}\left(r=R_{i}\right)=U, \\
& v_{\varphi}\left(r=R_{\mathrm{o}}\right)=0, \\
& v_{z}\left(r=R_{i}\right)=0,
\end{aligned}
$$


$v_{z}\left(r=R_{\mathrm{o}}\right)=0$,

The inner cylinder rotates at a constant angular velocity, $\boldsymbol{\Omega}=U / R_{\mathrm{i}} \mathbf{e}_{z}$, generating Couette flow in the annular gap and a constant pressure drop along the cylinder axis, $\nabla_{z} p$, leads to axial through-flow.

The Pompon equations are rendered dimensionless as before for the channel and tube flow problems, except that the characteristic length scale of the flow geometry now is, $\delta$, i.e., $\widetilde{\boldsymbol{r}}=\boldsymbol{r} / \delta, \widetilde{\boldsymbol{\nabla}}=\delta \boldsymbol{\nabla}$ Then, the Pompon Eqs. 1, 2, 3, 4 and 5 adopt the form

$$
\begin{aligned}
& \text { Ta } \mathbf{v} \cdot \boldsymbol{\nabla} \mathbf{v}=\boldsymbol{\nabla} \cdot\left(\lambda^{2} \mathbf{S}\right)-\boldsymbol{\nabla} p \\
& -\frac{v_{\varphi}}{r} \mathbf{M}^{i}+\mathbf{S} \cdot \mathbf{L}^{\mathrm{T}}+\mathbf{L} \cdot \mathbf{S} \\
& -\frac{1}{\left(1+a_{1}\right) \widetilde{\lambda}_{1}}\left\{3 \mathbf{S} \cdot \mathbf{S}+\left(a_{1}-1\right) \mathbf{S}-\frac{a_{1}}{3} \mathbf{1}\right\}=0,
\end{aligned}
$$

$\lambda \mathbf{L}: \mathbf{S}-\frac{1}{\widetilde{\lambda}_{2}}(\lambda-1) e^{\nu^{*}(\lambda-1)}=0$,

where Ta $=\rho^{2} U^{2} \delta^{3} /\left(R_{i} C^{2} g^{2} \lambda_{\mathrm{b}} \lambda_{\mathrm{s}}\right)$ is the Taylor number. The first term on the left side of Eq. 41 is obtained from the general relationship

$\mathbf{v} \cdot \nabla \mathbf{S}=\widehat{Q} \mathbf{S}+\frac{v_{\varphi}}{r} \mathbf{M}$

which holds for cylindrical coordinates (Tanner 2000). In Eq. 43, we have $\widehat{Q}=v_{r} \partial_{r}+v_{\varphi} / r \partial_{\varphi}+v_{z} \partial_{z}$ and

$\mathbf{M}=\left(\begin{array}{ccc}-2 S_{r \varphi} & S_{r r}-S_{\varphi \varphi} & -S_{\varphi z} \\ * & -2 S_{r \varphi} & S_{r z} \\ * & * & 0\end{array}\right)$,

is a matrix with $*$ denoting a symmetric entry. Note that for laminar flow in the Taylor-Couette geometry, we have $\widehat{Q}=0$ since $v_{r}=0$, and the flow is homogeneous in the $\mathbf{e}_{\varphi}$ and $\mathbf{e}_{z}$ direction. In the above Eqs. 40, 41 and 42, we have nonlinear convective terms in the momentum balance and in the backbone orientation tensor equation due to the rotation of the inner cylinder. These inertial terms are not present in laminar channel and tube flow with a stationary wall but in Couette flow between rotating cylinders. We will show sample calculations for the flow Eqs. 40, 41 and 42 for a specific choice of the relaxation times, parameter $a_{1}$, and various values of the number of Pompon arms. However, we present first the system equations for the TCMM Model.

The TCMM equations are rendered dimensionless with the characteristic length scale of the flow geometry $\delta$. Then, the model Eqs. 1, 2, 7, 8 and 9 adopt the form

$$
\mathrm{Ta} \mathbf{v} \cdot \boldsymbol{\nabla} \mathbf{v}=\sum_{i=1}^{2} \widetilde{G}_{i} \boldsymbol{\nabla} \cdot \mathbf{C}^{i}-\boldsymbol{\nabla} p
$$

$$
\begin{aligned}
-\frac{v_{\varphi}}{r} \mathbf{M}^{i} & +\mathbf{C}^{i} \cdot \mathbf{L}^{\mathrm{T}}+\mathbf{L} \cdot \mathbf{C}^{i}-\frac{1}{\widetilde{\lambda}_{i}} \mathbf{C}^{i} \\
& +\frac{1}{\widetilde{\lambda}_{i}} \mathbf{1}-\frac{\theta}{2 \widetilde{G}_{\mathrm{i}}}\left(\mathbf{C}^{1} \cdot \mathbf{C}^{2}+\mathbf{C}^{2} \cdot \mathbf{C}^{\mathbf{1}}-2 \mathbf{C}^{i}\right)=\mathbf{0}
\end{aligned}
$$

where we have introduced the Taylor number Ta $=\rho^{2} U^{2} \delta^{3} /$ $\left(R_{i} G_{1} \lambda_{1} G_{2} \lambda_{2}\right)$. The symmetric matrices $\mathbf{M}^{i}$ are defined in analogy to Eq. 44 for conformation tensor $\mathbf{C}^{i}(i=\{1,2\})$. Equations 45 and 46 are analogous to Eqs. 18 and 19 except that we now have inertial effects due to inner cylinder rotation. In the next section, the flow Eqs. 45 and 46 will be solved for vanishing coupling parameter, $\theta$, to compare with analytical results of the 2MUCM Model.

\section{Analytical solution for the 2MUCM model}

For the Taylor-Couette geometry with axial through-flow, the continuum equations (Eqs. 45 and 46) can be solved analytically for vanishing coupling parameter $(\theta=0)$. The analytical solution is developed as follows. First, we want to reformulate and solve the momentum balance equation for our flow problem. The $v_{r}$-component of the momentum balance (Eq. 45) gives the differential equation for the radial pressure distribution

$\frac{\partial p}{\partial r}-\sum_{i=1}^{2} \widetilde{G}_{i} \frac{\partial C_{r r}^{i}}{\partial r}=\mathrm{Ta} \frac{v_{\varphi}^{2}}{r}+\sum_{\mathrm{i}=1}^{2} \widetilde{G}_{\mathrm{i}}\left(\frac{C_{r r}^{i}}{r}-\frac{C_{\varphi \varphi}^{i}}{\mathrm{r}}\right)$,

and will be solved below after we have solved the conformation tensor (Eq. 46). For the $v_{\varphi}$-component of the momentum balance (Eq. 45), we have 
$r^{2} \frac{\partial^{2} v_{\varphi}}{\partial r^{2}}+r \frac{\partial v_{\varphi}}{\partial r}-v_{\varphi}=0$

which is obtained upon insertion of the $C_{r \varphi}^{i}$ components of Eq. 46 into the $v_{\varphi}$-component of Eq. 45 . The general solution of this differential equation is

$v_{\varphi}=k_{-1} \frac{1}{r}-k_{1} r$

where $k_{-1}$ and $k_{1}$ are constants to be determined from the boundary conditions. With Eqs. 36 and 37, we obtain the following azimuthal velocity field

$v_{\varphi}=\frac{R_{i} U}{R_{\mathrm{o}}^{2}-R_{\mathrm{i}}^{2}}\left(R_{\mathrm{o}}^{2} \frac{1}{r}-r\right)$,

$v_{\varphi}^{\prime}=-\frac{R_{i} U}{R_{\mathrm{o}}^{2}-R_{\mathrm{i}}^{2}}\left(R_{\mathrm{o}}^{2} \frac{1}{r^{2}}+1\right)$

The $v_{z}$-component of the momentum balance (Eq. 18) is equivalent to

$\widetilde{\eta} r \frac{\partial^{2} v_{z}}{\partial r^{2}}+\widetilde{\eta} \frac{\partial v_{z}}{\partial r}=r \frac{\partial p}{\partial z}$.

Equation 52 is obtained by inserting the $C_{r z}^{i}$-components of the conformation tensor (Eq. 46) into the momentum balance for $v_{z}$, (Eq. 45). The general solution of Eq. 52 is

$v_{z}=\frac{1}{4 \widetilde{\eta}} \frac{\partial p}{\partial z} r^{2}+k_{l} \ln r+k_{0}$,

where $k_{l}$ and $k_{0}$ are constants which, again, have to be determined from the boundary conditions. From the boundary conditions of Eqs. 38 and 39, we get

$v_{z}=-\frac{1}{4 \widetilde{\eta}} \frac{\partial p}{\partial z}\left[\left(R_{\mathrm{o}}^{2}-R_{\mathrm{i}}^{2}\right) \frac{\ln \left(\frac{r}{R_{i}}\right)}{\ln \left(\frac{R_{\mathrm{o}}}{R_{\mathrm{i}}}\right)}-\left(r^{2}-R_{i}^{2}\right)\right]$,

$v_{z}^{\prime}=-\frac{1}{2 \widetilde{\eta}} \frac{\partial p}{\partial z}\left(\frac{R_{\mathrm{o}}^{2}-R_{\mathrm{i}}^{2}}{2 \ln \left(\frac{R_{\mathrm{o}}}{R_{\mathrm{i}}}\right)} \frac{1}{r}-r\right)$.

With Eqs. 51 and 55, we can now solve the conformation tensor (Eq. 46) to obtain

$C_{r r}^{i}=1$,

$$
C_{r \varphi}^{i}=-\widetilde{\lambda}_{i} \frac{2 R_{\mathrm{o}}^{2} R_{\mathrm{i}} U}{R_{\mathrm{o}}^{2}-R_{\mathrm{i}}^{2}} \frac{1}{r^{2}},
$$

$C_{r z}^{i}=-\frac{\widetilde{\lambda}_{i}}{2 \widetilde{\eta}}\left(\frac{R_{\mathrm{o}}^{2}-R_{\mathrm{i}}^{2}}{2 \ln \left(\frac{R_{\mathrm{o}}}{R_{\mathrm{i}}}\right)} \frac{1}{r}-r\right) \frac{\partial p}{\partial z}$,

$C_{\varphi \varphi}^{i}=2 \widetilde{\lambda}_{i}^{2}\left(-\frac{2 R_{\mathrm{o}}^{2} R_{\mathrm{i}} U}{R_{\mathrm{o}}^{2}-R_{\mathrm{i}}^{2}} \frac{1}{r^{2}}\right)^{2}+1$

$C_{\varphi z}^{i}=2 \frac{\widetilde{\lambda}_{i}^{2}}{\widetilde{\eta}} \frac{R_{\mathrm{o}}^{2} R_{\mathrm{i}} U}{R_{\mathrm{o}}^{2}-R_{\mathrm{i}}^{2}} \frac{1}{r^{2}}\left(\frac{R_{\mathrm{o}}^{2}-R_{\mathrm{i}}^{2}}{2 \ln \left(\frac{R_{\mathrm{o}}}{R_{\mathrm{i}}}\right)} \frac{1}{r}-r\right) \frac{\partial p}{\partial z}$,

$C_{z z}^{i}=2 \widetilde{\lambda}_{i}^{2}\left[-\frac{1}{2 \widetilde{\eta}}\left(\frac{R_{\mathrm{o}}^{2}-R_{\mathrm{i}}^{2}}{2 \ln \left(\frac{R_{\mathrm{o}}}{R_{\mathrm{i}}}\right)} \frac{1}{r}-r\right) \frac{\partial p}{\partial z}\right]^{2}+1$,

for the radial normal conformations $C_{r r}^{i}$, the radial angular shear conformations due to rotation $C_{r \varphi}^{i}$, the radial axial shear conformations $C_{r z}^{i}$, the angular normal conformations $C_{\varphi \varphi}^{i}$, the angular axial shear conformations $C_{\varphi z}^{i}$, and the axial normal conformations $C_{z z}^{i}$ for $i=\{1,2\}$. The corresponding extra stress tensor components are obtained from Eq. 9. Finally, with Eqs. 56 and 59, we can solve the $v_{r^{-}}$ component of the momentum equation which gives the radial pressure distribution

$$
\begin{aligned}
p= & \mathrm{Ta}\left(\frac{R_{\mathrm{i}} U}{R_{\mathrm{o}}^{2}-R_{\mathrm{i}}^{2}}\right)^{2} \times \\
& {\left[\frac{1}{2} R_{\mathrm{o}}^{4}\left(\frac{1}{R_{\mathrm{i}}^{2}}-\frac{1}{r^{2}}\right)+2 R_{\mathrm{o}}^{2} \ln \left(\frac{R_{\mathrm{i}}}{r}\right)-\frac{1}{2}\left(R_{\mathrm{i}}^{2}-r^{2}\right)\right] } \\
& -\frac{1}{2} \widetilde{\eta}_{2}\left(\frac{2 R_{\mathrm{o}}^{2} R_{\mathrm{i}} U}{R_{\mathrm{o}}^{2}-R_{\mathrm{i}}^{2}}\right)^{2}\left(\frac{1}{R_{\mathrm{i}}^{4}}-\frac{1}{r^{4}}\right) .
\end{aligned}
$$


The volumetric flow rate through the annular gap is

$$
\begin{aligned}
Q & =\int_{0}^{2 \pi} d \varphi \int_{R_{i}}^{R_{o}} r v_{z}(r) \mathrm{d} r \\
& =-\frac{\pi}{8 \widetilde{\eta}}\left(R_{\mathrm{o}}^{4}-R_{\mathrm{i}}^{4}\right)\left(1-\frac{1}{\ln \left(\frac{R_{\mathrm{o}}}{R_{\mathrm{i}}}\right)} \frac{R_{\mathrm{o}}^{2}-R_{\mathrm{i}}^{2}}{R_{\mathrm{o}}^{2}+R_{\mathrm{i}}^{2}}\right),
\end{aligned}
$$

and the average axial velocity is given as $\bar{V}=Q /$ $\left(\pi\left(R_{\mathrm{o}}^{2}-R_{\mathrm{i}}^{2}\right)\right)$. In what follows, we explain the numerical scheme that we have adopted to solve the Pompon equations and the TCMM equations for laminar Taylor-Couette flow.
Numerical method

The Pompon Eqs. 40, 41 and 42 can be reformulated in terms of a TPBV problem, Eq. 29, for the radial coordinate and the boundary conditions (Eqs. 36, 37, 38 and 39). The 12-tuple of flow profiles to solve for is

$\boldsymbol{\xi}=\left(p, v_{\varphi}, v_{\varphi}^{\prime}, v_{z}, v_{z}^{\prime}, \operatorname{vec}(\mathbf{S}), \lambda\right)^{T}$,

where $\operatorname{vec}(\mathbf{S})=\left(S_{r r}, S_{r \varphi}, S_{r z}, S_{\varphi \varphi}, S_{\varphi z}, S_{z z}\right)$. The $12 \times 12$ coefficient matrix, $\mathcal{A}$, and the inhomogeneity, b, in Eq. 29 are obtained as for the laminar channel and tube flow problem in the previous sections. Note that we have here to match two boundary conditions on the outer cylinder, Eqs. 37 and 39. Therefore, a two-dimensional Newton method for the variables $v_{\varphi}^{\prime}$ and $v_{z}^{\prime}$ is embedded into the shooting algorithm to match the two boundary conditions, Eqs. 37 and 39 .
Fig. 9 Laminar annular flow profiles $(\kappa=0.5)$ of a Pompon fluid for five inner cylinder velocities: $U=2$ (solid lines), 4 (dotted lines), 6 (dashed lines), 8 (long-dashed lines), and 10 (dot-dashed lines), and $\nabla_{z} p=0$. Model parameters are $\lambda_{\mathrm{b}} / \lambda_{\mathrm{s}}=3$, $a_{1}=1$, and $q=3$. a Azimuthal velocity, $\mathbf{b}$ shear rate, $\mathbf{c}$ radial normal stress, d azimuthal normal stress, e radial azimuthal shear stress, and f backbone stretch
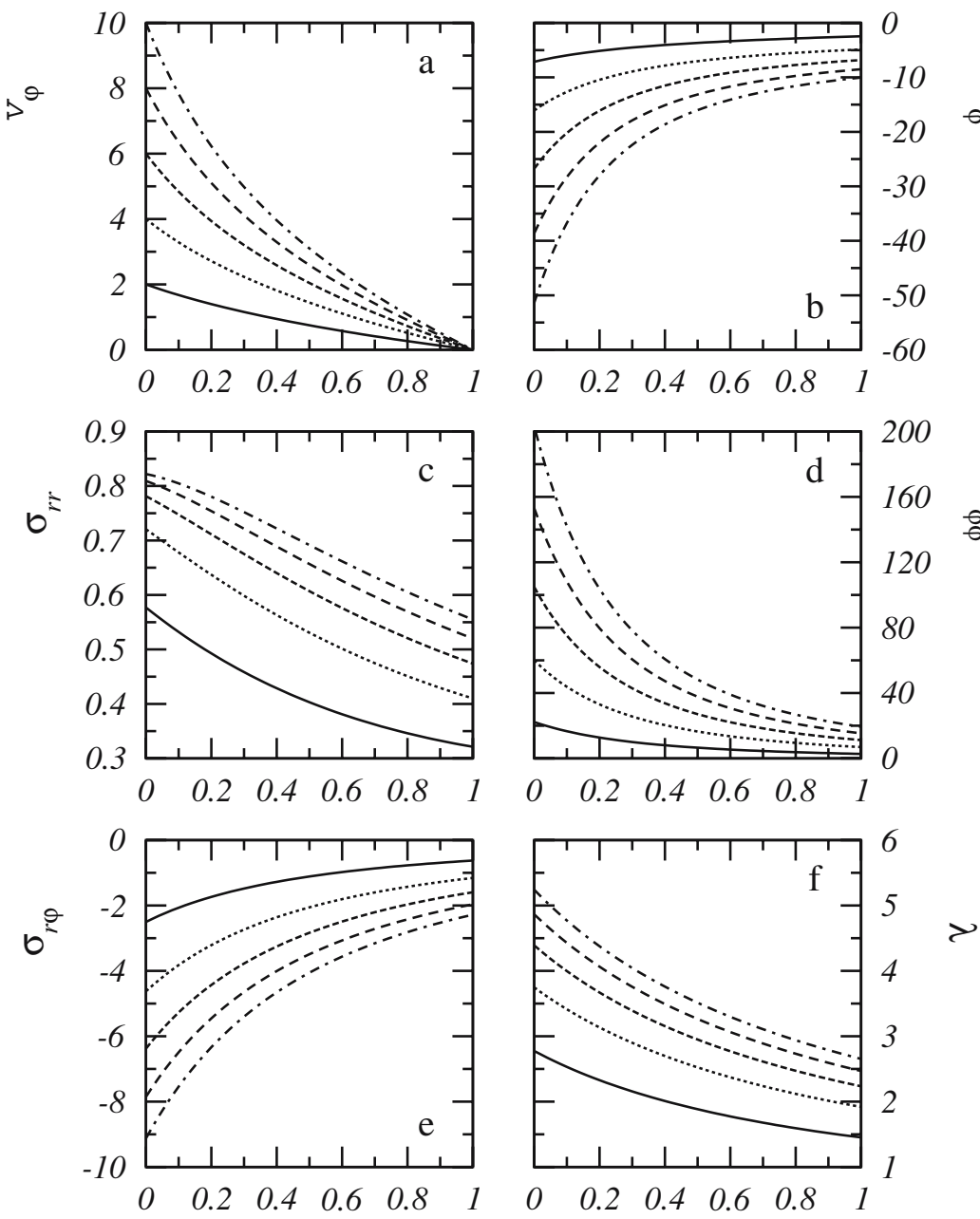

$\frac{r-R_{\mathrm{i}}}{R_{\mathrm{o}}-R_{\mathrm{i}}}$
6

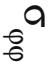


Consequently, before every Runge-Kutta integration in the shooting algorithm, the homogeneous shear flow problem for the velocity gradient

$\boldsymbol{\nabla} \mathbf{v}=\left(\begin{array}{ccc}0 & -\frac{v_{\varphi}}{r} & 0 \\ \frac{\partial v_{\varphi}}{\partial r} & 0 & 0 \\ \frac{\partial v_{z}}{\partial r} & 0 & 0\end{array}\right)$,

has to be solved on the inner cylinder to determine correct starting values for the structural variables $\mathbf{S}$ and $\lambda$ on the inner cylinder.

For laminar Taylor-Couette flow of a TCMM fluid, the system Eqs. 45 and 46 are reformulated in terms of a TPBV problem, Eq. 29, in analogy to the Pompon Model. The 17tuple of unknowns is now

$\boldsymbol{\xi}=\left(p, v_{\varphi}, v_{\varphi}^{\prime}, v_{z}, v_{z}^{\prime}, \operatorname{vec}\left(\mathbf{C}^{1}\right), \operatorname{vec}\left(\mathbf{C}^{2}\right)\right)^{\mathrm{T}}$,

where $\operatorname{vec}\left(\mathbf{C}^{i}\right)=\left(C_{r r}^{i}, C_{r \varphi}^{i}, C_{r z}^{i}, C_{\varphi \varphi}^{i}, C_{\varphi z}^{i}, C_{z z}^{i}\right), \quad i=$ $\{1,2\}$. The $17 \times 17$-coefficient matrix, $\mathcal{A}$, and the inhomogeneity, $\mathbf{b}$, in Eq. 29 are obtained as in the previous sections and the boundary conditions (Eqs. 36, 37, 38 and 39) are the same as for the Pompon Model. In what follows, we present sample calculations for the Pompon Model and the TCMM Model in the Taylor-Couette geometry with and without axial through-flow and inner cylinder rotation.
Fig. 10 Laminar annular flow $(\kappa=0.5)$ profiles of a Pompon fluid for five pressure drops $\nabla_{z} p=-2$ (solid lines), -4 (dotted lines), -6 (dashed lines), -8 (long-dashed lines), and -10 (dot-dashed lines), and $U=0$. Model parameters are $\lambda_{\mathrm{b}} / \lambda_{\mathrm{s}}=3$, $a_{1}=1$, and $q=3$. a Axial velocity, $\mathbf{b}$ shear rate, $\mathbf{c}$ radial normal stress, $\mathbf{d}$ radial axial stress, e axial normal stress, and $\mathbf{f}$ backbone stretch
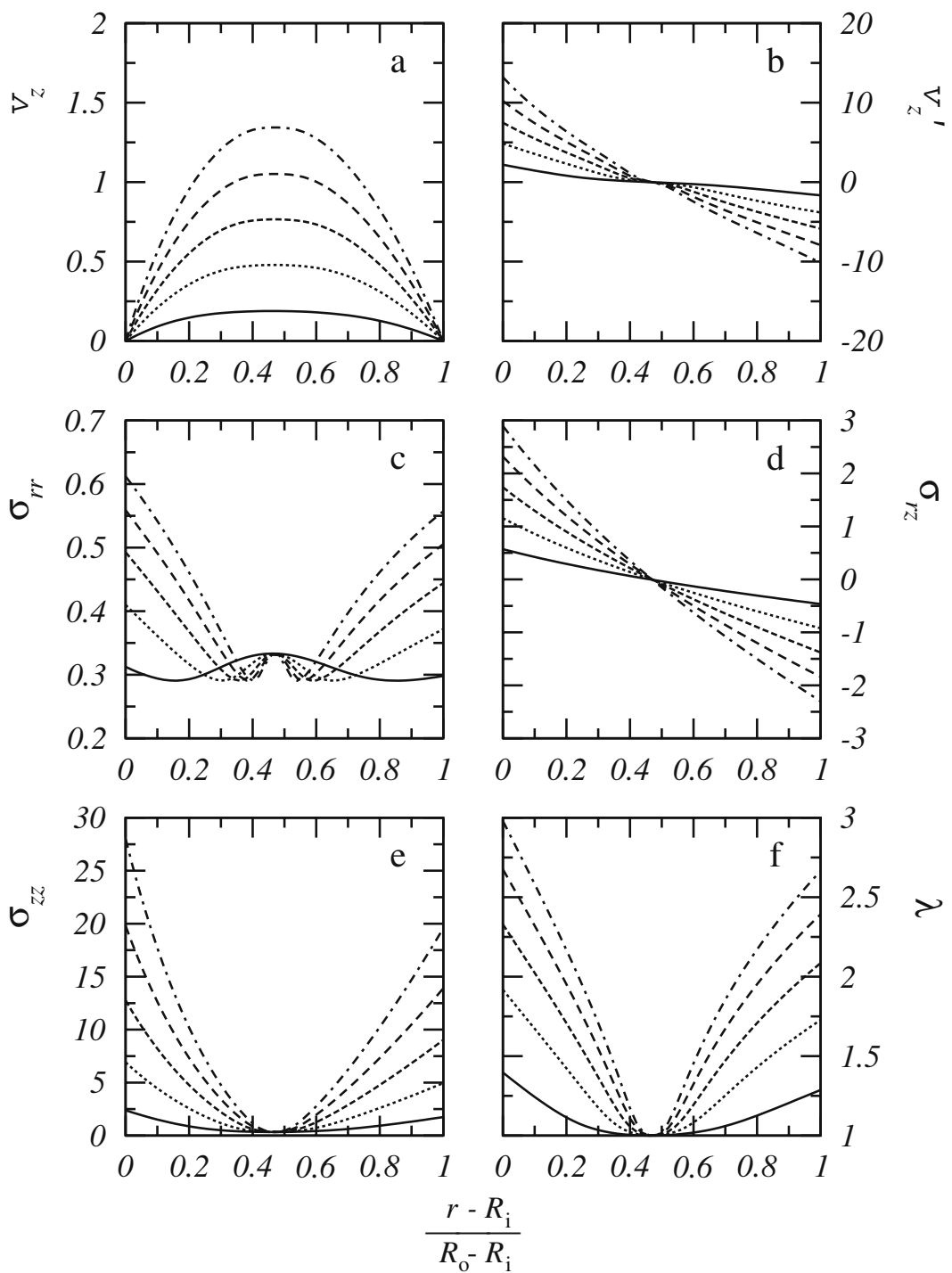
Sample calculations

\section{The Pompon model}

The boundary value problem for annular flow of a Pompon fluid between concentric cylinders $(\kappa=0.5)$ has been solved for $\lambda_{\mathrm{b}} / \lambda_{\mathrm{s}}=3, a_{1}=1$, and different values of the number of arms, $q$. Sample calculations are reported in Figs. 9, 10, 11, 12,13 and 14. In what follows, we report model predictions for Couette flow due to inner cylinder rotation (Fig. 9), for pressure-driven axial flow between stationary concentric cylinders (Fig. 10), and for combined Poiseuille and Couette flow (Figs. 11, 12, 13 and 14).

In Fig. 9, we display model predictions for Couette flow between concentric cylinders where the inner cylinder is rotating with a constant velocity $U=v_{\varphi}(\bar{r}=0)$. We report sample calculations for five inner cylinder velocities, which illustrate that the wall shear rate (Fig. 9b), the extra stresses (Fig. 9e), and the backbone stretch (Fig. 9f) are larger on the inner cylinder than on the outer cylinder. Figure 9a displays the angular velocity as a function of the relative gap
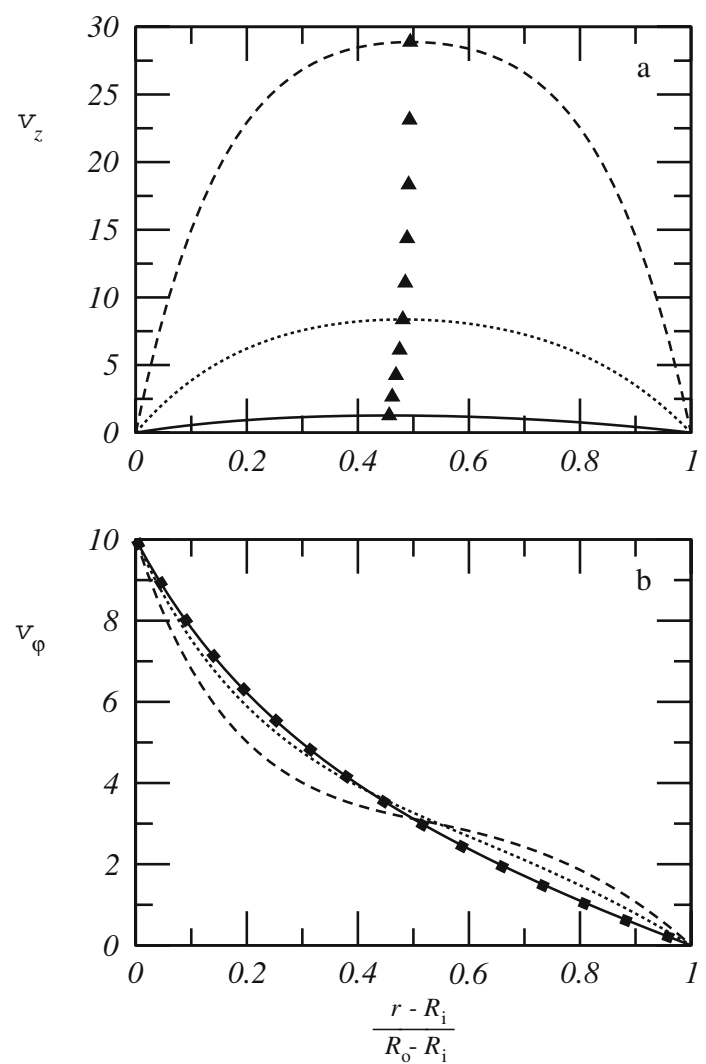

Fig. 11 Axial (a) and azimuthal (b) velocity profiles for TaylorCouette flow $(\kappa=0.5)$ of a Pompon fluid with inner cylinder velocity $U=10$ and three axial pressure drops: $\nabla_{z} p=-10$ (solid lines), -50 (dotted lines), and -100 (dotted lines). Model parameters are $\lambda_{\mathrm{b}} / \lambda_{\mathrm{s}}=3, a_{1}=1$, and $q=3$. The dots in $\mathbf{b}$ are for $\nabla_{z} p=0$ distance. The angular velocity is $U$ at the inner cylinder and it vanishes on the outer cylinder. The corresponding shear rate is displayed in Fig. 9b, where we notice that the variation of the local shear rate is stronger near the inner cylinder than near the outer cylinder. In Fig. 9c,d, we display the radial and the angular normal stress distributions in the annular gap, respectively. Both normal stresses are positive; they decrease monotonically as a function of the relative position in the gap, and they increase as a function of inner cylinder velocity, $U$. Note that the radial normal stresses are smaller than the angular normal stresses. The radial angular shear stress shown in Fig. 9e correlates with the shear rate shown in Fig. 9b. The backbone stretch profiles for the five inner cylinder rotations are shown in Fig. 9f. The backbone stretch decreases monotonically between the two cylinders and it increases as the inner cylinder velocity increases. The corresponding results for the TCMM Model are shown in Fig. 15 and will be discussed below.

Figure 10 displays the properties of the Pompon Model for axial-pressure-driven flow between concentric cylinders and $U=0$. We display sample calculations for five pressure drops which, again, illustrate that the shear rate (Fig. 10b), extra stresses (Fig. 10c-e), and backbone stretch (Fig. 10f) are more elevated on the inner cylinder than on the outer one. The axial velocity in the cylindrical gap (Fig. 10a) vanishes on the two cylinders, as required by the no-slip boundary conditions, and it has a maximum in between. The corresponding shear rate profile is shown in Fig. 10b, where we notice that the wall shear rates are higher on the inner cylinder than on the outer one.

The extra stress tensor profiles are displayed in Fig. 10c-e. Remember that the stress tensor of the Pompon fluid is $\sigma=1 / 3$ for vanishing velocity gradient. Figure 10c shows the radial normal stress profiles, which behave qualitatively different compared to pure Couette flow (cf. Fig. 9c) as they oscillate in the central area of the gap. The highest value of the radial normal stress is found on the inner cylinder. The radial axial shear-stress profile in Fig. 10d correlates with the shear-rate profile shown in Fig. 10b. The axial normal-stress profile in Fig. 10e is positive and two orders of magnitude larger than the radial normal stresses. The backbone stretch profiles for the five pressure drops in Fig. 10f have a maximum on the inner cylinder, followed by a decrease to the equilibrium value, $\lambda=1$, where the shear rate vanishes, and an increase as the outer cylinder is approached. The corresponding results for the TCMM Model is presented in Fig. 16.

In Figs. 11, 12, 13 and 14, we summarize the flow profiles for mixed Couette and Poiseuille flow between concentric cylinders. We report sample calculations for inner cylinder velocity, $U=10$, and three axial pressure drops, $\nabla_{z} p=-10$, -50 , and -100 . Pompon Model parameters are the same as before, in Figs. 9 and 10.

Figure 11 displays the axial (Fig. 11a) and azimuthal fluid velocities (Fig. 11b) in the annular gap. The axial velocity vanishes on the boundaries, and it has a maximum 
Fig. 12 Extra stress tensor profiles for laminar Taylor-Couette flow of a Pompon fluid for $U=10$ and three axial pressure drops: $\nabla_{z} p=-10$ (solid lines), -50 (dotted lines), and -100 (dashed lines). Model parameters and geometry parameters are the same as in Fig. 9
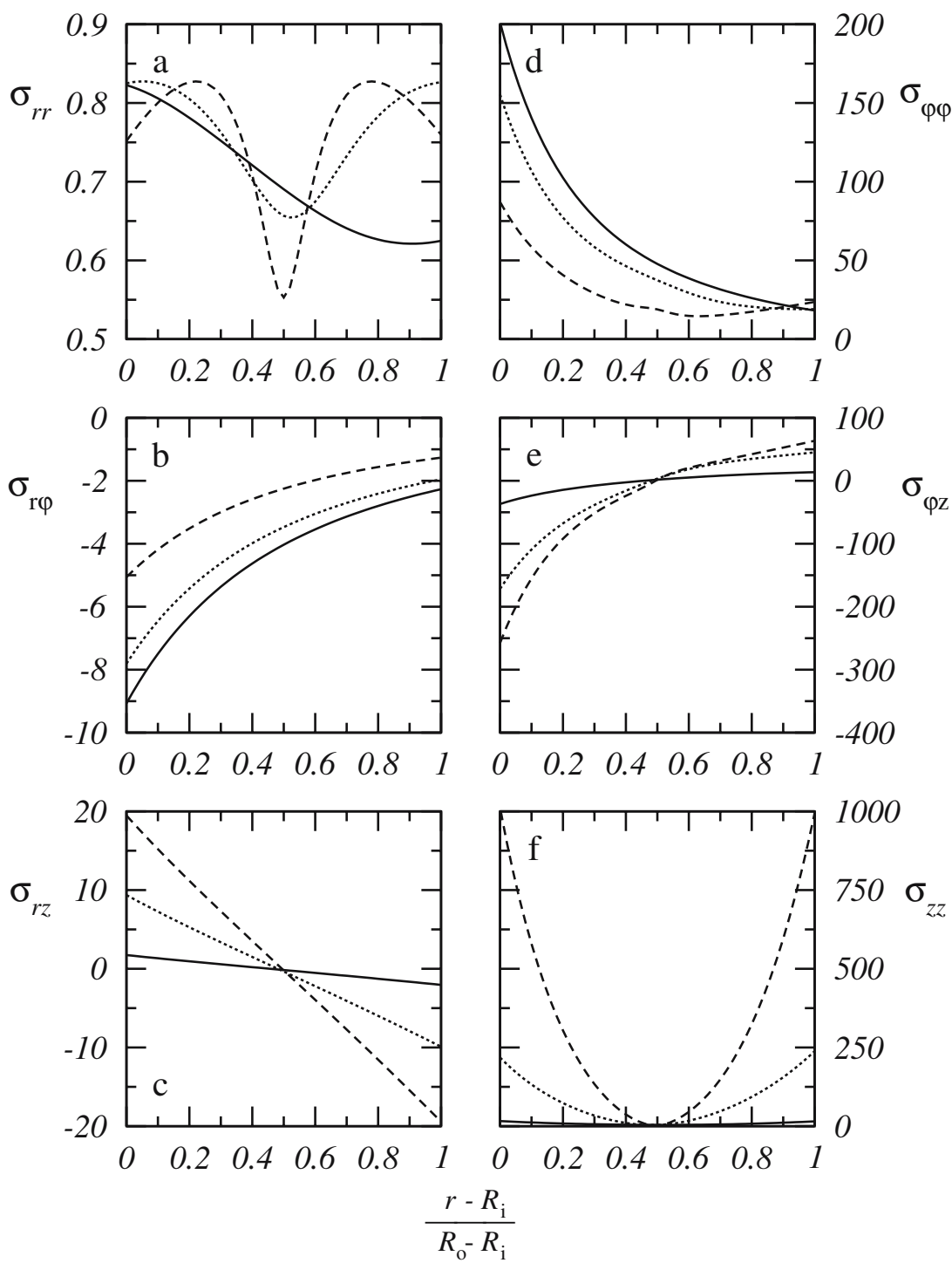

value between $\bar{r}=0.4$ and 0.5 . The solid triangles in Fig. 11a mark the position of maximum axial fluid velocity for ten pressure drops between $\nabla_{z} p=-10$ and -100 . They have been determined numerically with a "golden section" search in one dimension (Press et al. 1992). Figure 11b displays the corresponding angular velocity profiles. The angular velocity is $v_{\varphi}=10$ on the inner cylinder, and it vanishes on the outer cylinder as required by the boundary conditions Eqs. 36 and 37. A high axial pressure drop (dashed line in Fig. 11b) leads to a decrease of the angular velocity near the inner cylinder and to an increase of the angular velocity near the outer cylinder with respect to the flow field for zero pressure drop (dots in Fig. 11b).

The components of the extra stress tensor corresponding to the velocity field in Fig. 11 are shown in Fig. 12. Figure 12a displays the radial normal stress profiles for the three pressure drops. This extra stress tensor component is small (in comparison with the other two normal stress components in Fig. 2d,f) and positive. For the smallest pressure drop, it is almost monotonic as found for pure Couette flow (cf. Fig. 9c). For the largest pressure drop, it is oscillating as observed for axial annular flow (cf. Fig. 10c). Note that for large pressure drops, the position of maximum radial normal stress is not on the boundary but in the gap.

The radial angular shear stress and the radial axial shear stress are shown in Fig. 12b,c, respectively. Both shear stresses are monotonic functions, and they correlate with the respective shear rate profiles. Note that the absolute magnitude of the radial angular shear stress decreases with increasing pressure drop and constant inner cylinder velocity.

Figure $12 \mathrm{~d}$ displays the angular normal stress profiles in the annular gap which are monotonic for the small pressure drops. For the large pressure drops, they are non- 




Fig. 13 The same as in Fig. 12 for the backbone stretch variable

monotonic near the outer cylinder. Similarly as for the absolute value of the radial angular shear stress, the angular normal stresses also decrease as the pressure drop increases and the inner cylinder velocity remains constant.

Figure 12e displays the angular axial shear stress profiles. This extra stress tensor component is only found in mixed Couette and Poiseuille flow and it has a maximum absolute value on the inner cylinder which increases with increasing pressure drop. The axial normal-stress profiles are shown in Fig. 12f. Note that for a pressure drop $\nabla_{z} p=-50$, the maximum axial normal stress is found on the outer cylinder.

Figure 13 displays the profile of the backbone stretch in the annular gap. We see a transition of the backbone stretch profile from the flow regime dominated by Couette flow (for small axial pressure drops) to a flow regime dominated by Poiseuille flow (for large axial pressure drops). For small pressure drops, the backbone stretch profile is almost monotonic as we have found for pure Couette flow (solid line in Fig. 13). For large pressure drops, the backbone stretch profile is similar as for pure Poiseuille flow between concentric cylinders (dashed line in Fig. 13), i.e., it has a minimum with a value larger than unity due to the Couette flow component.

Figure 14 shows the maximum axial fluid velocity (Fig. 14a), the relative position of maximum fluid velocity (Fig. 14b), and the volumetric flow rate (Fig. 14c) as functions of the axial pressure drop. We report sample calculations for three numbers of arms, $q$. Note that the maximum fluid velocity (Fig. 14a) and the volumetric flow rate (Fig. 14c) decrease as the number of arms increases. This is qualitatively similar to the behavior found for tube flow of a Pompon fluid (Fig. 4c). The relative position of maximum fluid velocity in the axial direction has been


Fig. 14 Maximum fluid velocity in the annulus (a), relative position of maximum fluid velocity (b), and volumetric flow rate (c) for laminar Taylor-Couette flow of a Pompon fluid for $U=10$ and $q=3$ (solid lines), 6 (dotted lines), 9 (dashed lines). Model and geometry parameters are the same as in Fig. 11

determined with a golden section scheme, and it is found to lie between 0.4 and 0.55 .

\section{The TCMM model}

In Figs. 15, 16, 17, and 18, we report flow characteristics of the TCMM fluid for $\lambda_{2} / \lambda_{1}=2, n_{2} / n_{1}=1$ and various values of the coupling parameter. We investigate pure Couette flow, pure Poiseuille flow, and a combination of both in the annular gap between concentric cylinders. For our sample calculations, we use cylinder radii $R_{\mathrm{i}}=0.5$ and $R_{\mathrm{o}}=1$, i.e., $\kappa=0.5$.

Figure 15 displays the velocity (Fig. 15a,b), stress tensor (Fig. $15 \mathrm{c}-\mathrm{e}$ ), and pressure profiles (Fig. 15f) in the annular gap for five inner cylinder velocities and vanishing pressure drop, i.e., $v_{z}(r)=0$. The magnitude of the inner cylinder velocity can be identified from the abscissa of Fig. 15a. Note that, for the smallest value of inner cylinder velocity, the 
Fig. 15 Laminar annular flow $(\kappa=0.5)$ profiles of a TCMM fluid for five inner cylinder velocities: $U=2$ (solid lines), 4 (dotted lines), 6 (dashed lines), 8 (long-dashed lines), and 10 (dot-dashed lines), and $\nabla_{z} p=0$. a Azimuthal velocity, b velocity gradient, c radial normal stress, d azimuthal normal stress, e radial azimuthal shear stress, and $\mathbf{f}$ pressure. TCMM parameters are the same as in Fig. 6
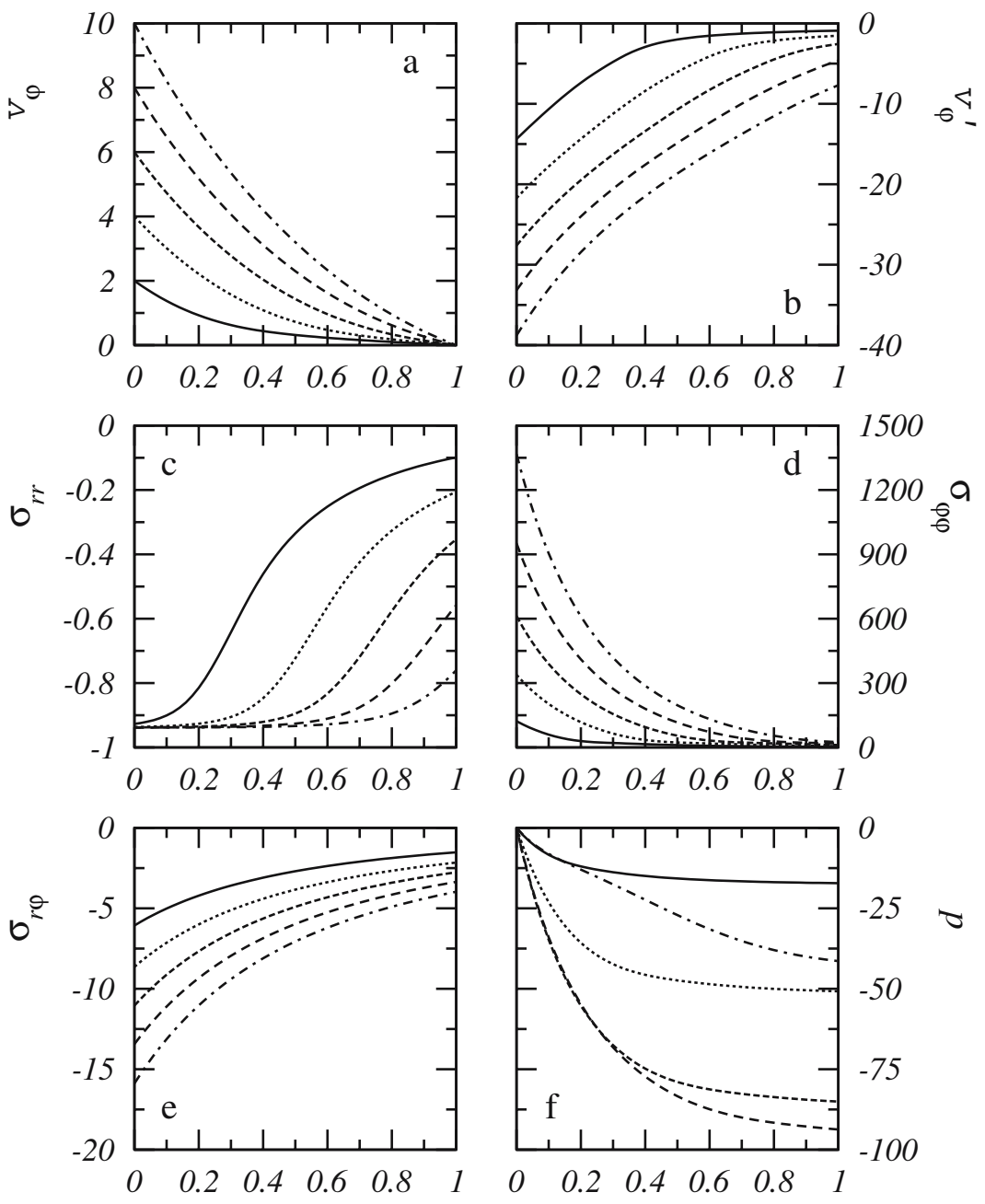

$\frac{r-R_{\mathrm{i}}}{R_{\mathrm{o}}-R_{\mathrm{i}}}$ velocity gradient in Fig. 15b increases strongly near the inner cylinder and reaches a plateau near the outer cylinder. The absolute magnitude of the angular shear rate decreases when going from the inner to the outer cylinder.

Figure $15 \mathrm{c}-\mathrm{f}$ show the stress tensor and pressure profiles corresponding to the velocity field in Fig. 15a,b. Note that the absolute values of the radial normal stresses (Fig. 15c) and the angular normal stresses (Fig. 15d) decrease as the outer cylinder is approached. The radial normal stresses in Fig. $15 \mathrm{c}$ are small and negative, being qualitatively different from the Pompon Model in Fig. 9c which predicts positive radial normal stresses. The angular normal stresses in Fig. 15d are large and positive and show the same qualitative behavior as for the Pompon Model (cf. Fig. 9). The radial angular shear stress (Fig. 15e) correlates with the behavior of the angular shear rate in Fig. 15b. The magnitude of the shear stress is large on the inner cylinder and small on the outer cylinder. We again obtain similar qualitative behavior as for the Pompon Model (cf. Fig. 9). The absolute magnitudes of the stresses in Fig. 15c-e are large at the inner cylinder and small at the outer cylinder.

The pressure profiles for laminar Couette flow of a TCMM fluid between concentric cylinders are reported in Fig. 15f. Note that the pressure on the outer cylinder as a function of inner cylinder velocity shows a non-monotonic behavior: it decreases for small inner cylinder velocities and it increases for large inner cylinder velocities.

Figure 16 displays annular flow profiles of the TCMM Model for axial-pressure-driven flow between concentric cylinders and five pressure drops. For the velocity field (Fig. 16a,b), the radial axial shear stress (Fig. 16d) and the axial normal stresses (Fig. 16e), we find qualitatively similar results as for the Pompon Model (cf. Fig. 10). The axial velocity in Fig. 16a vanishes on the walls as required by the no-slip boundary conditions, and it assumes a maximum value in between the two cylinders. The corresponding 
Fig. 16 Laminar annular flow $(\kappa=0.5)$ profiles of a TCMM fluid for five pressure drops: $\nabla_{z} p=-2$ (solid lines), -4 (dotted lines), -6 (dashed lines), -8 (long-dashed lines), and -10 (dot-dashed lines), and stationary cylinders, $U=0$. a Axial velocity, b velocity gradient, c radial normal stress, $\mathbf{d}$ radial axial shear stress, e axial normal stress, and f pressure. TCMM parameters are the same as in Fig. 6
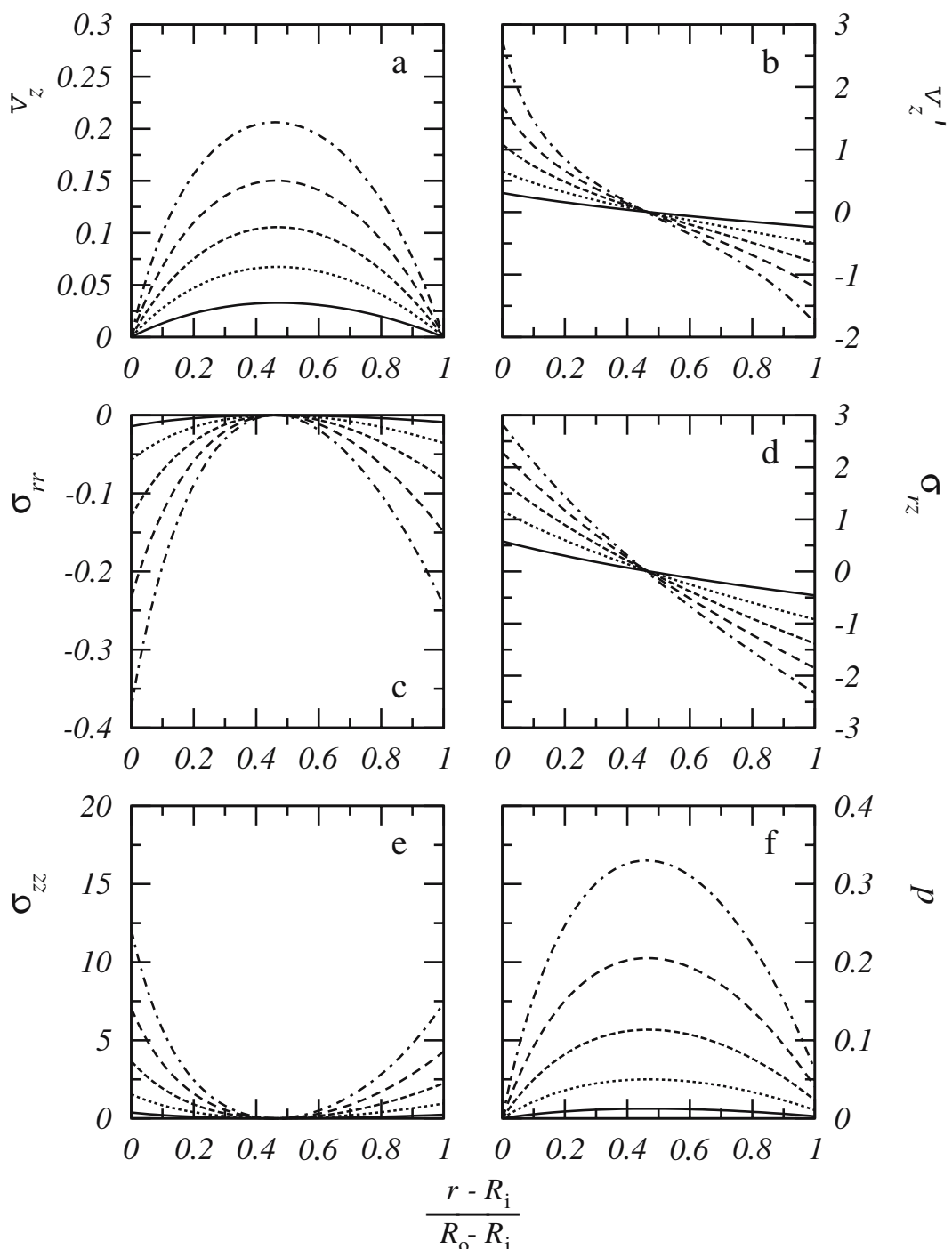

shear-rate profile is displayed in Fig. 16b where we notice that the wall shear rates are higher on the inner cylinder than on the outer one, similar to the Pompon Model (cf. Fig 10b).

The extra stress tensor profiles are displayed in Fig. 10c-e. For vanishing velocity gradient, the extra stresses of the TCMM fluid vanish, $\boldsymbol{\sigma}=0$. Figure $16 \mathrm{c}$ shows the radial normal stress profiles, which behave qualitatively differently than the Pompon Model (cf. Fig. 10c). The TCMM Model predicts negative radial normal stresses; the Pompon Model predicts positive radial normal stresses. The largest radial normal stresses (absolute values) for the TCMM fluid are found on the inner cylinder, which is the same as for the Pompon Model. The radial axial shear stress profile of Poiseuille flow between concentric cylinders is shown in Fig. 16d. We find that this quantity correlates with the shearrate profile shown in Fig. 16b, and that it has its maximum value on the inner cylinder, too. The axial normal-stress profiles of the TCMM Model (Fig. 16e) are positive and larger than the absolute value of the radial normal stresses. The radial pressure distribution for axial annular flow of a TCMM fluid is shown in Fig. 16f. This quantity has an absolute maximum in the middle of the annular gap and a non-trivial value on the outer cylinder.

In Figs. 17 and 18, we show flow profiles for mixed Couette and Poiseuille flow of a TCMM fluid between concentric cylinders. Again, we report sample calculations for inner cylinder velocity $U=10$ and three axial pressure drops: $\nabla_{z} p=-10,-50$, and -100 . TCMM Model parameters are as before in Figs. 15 and 16.

Figure 17 displays the axial (Fig. 17a) and the azimuthal (Fig. 17b) fluid velocity in the annulus. The axial velocity field fulfills the no-slip boundary conditions Eqs. 38 and 39 on the two cylinders, and it has a maximum in between. The solid triangles in Fig. 17a show the maximum axial fluid velocity for ten pressure drops between $\nabla_{z} p=-10$ and -100 , which have been calculated with a golden section 

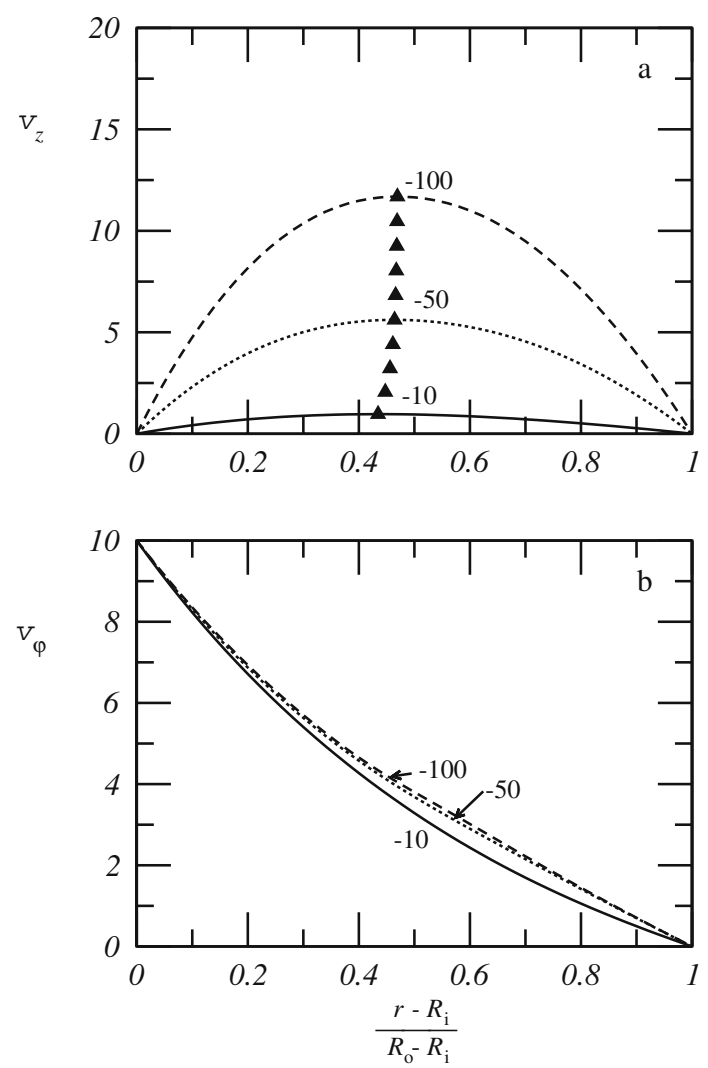

Fig. 17 Velocity profiles in the gap, $\kappa=0.5$, for three axial pressure drops: $\nabla_{z} p=-10$ (solid lines), -50 (dotted lines), and -100 (dashed lines). The velocity of the inner cylinder is $U=10$. a Axial velocity and $\mathbf{b}$ azimuthal velocity. Model parameters are the same as in Fig. 6

search. Figure $17 \mathrm{~b}$ displays the corresponding angular flow profiles that fulfill the boundary conditions Eqs. 36 and 37. Note that the axial pressure drop increases the angular fluid velocity in the gap, which is qualitatively different from the sample calculations for the Pompon Model (cf. Fig. 11b). For the Pompon Model, we found a decrease of the angular velocity near the inner cylinder and an increase near the outer cylinder (with respect to pure Couette flow) due to the applied pressure drop.

The extra stress-tensor profiles corresponding to the velocity field in Fig. 17 are reported in Fig. 18. Figure 18a shows the radial normal stress profiles in the gap, which are small and negative. For the smallest pressure drop, the radial normal stresses are dominated by the inner cylinder rotation, and we observe a monotonically increasing radial normal stress profile (cf. Fig. 15c). For large pressure drops, i.e., a flow which is dominated by the axial pressure drop, the radial normal stress has a maximum in the middle of the annular gap and is similar to the profile found in pure Poiseuille flow (cf. Fig. 16c). The radial angular shear stress (Fig. 18b) and the radial axial shear stress (Fig. 18c) correlate with the axial shear rate and the angular shear rate, respectively. The absolute magnitude of the radial angular shear stress decreases as the pressure drop increases, and the variations of the radial axial shear stress become more pronounced as the pressure drop is increased. This behavior is qualitatively similar to that observed for the Pompon Model (cf. Fig. 12b,c).

The angular normal stresses are displayed in Fig. 18d. These stresses are large and positive, and vary slightly with the pressure drop. Note that the angular normal stresses on the inner cylinder decrease, whereas they increase on the outer cylinder as the pressure drop is increased. This is qualitatively similar to the results found for the Pompon Model (cf. Fig. 12d).

The angular axial shear-stress and the axial normal-stress profiles are displayed in Fig. 18e,f, respectively. The angular axial shear stresses become more important as the pressure drop is increased. The absolute value of this shear stress component is larger on the inner cylinder than on the outer cylinder. The same behavior has also been found for the Pompon Model (Fig. 12e). The axial normal stress profiles are non-monotonic, and they assume their maxima on the inner cylinder. This is qualitatively different than that observed for the Pompon Model (cf. Fig. 12f).

\section{Conclusions}

In the present article, the Pompon, Pom-Pom, and TCMM models for viscoelastic fluids have been solved for laminar channel, tube, and annular flow with and without axial through-flow to compute laminar flow profiles in these geometries. The dynamic equations for the three models have been formulated in terms of TPBV problems, and several modifications of a shooting algorithm have been adapted to solve the continuum equations in the three flow geometries. Furthermore, analytical solutions for the 2MUCM Model have been derived to verify the consistency of the numerical codes for the TCMM equations with the analytical solutions. The velocity, backbone stretch, stress tensors, and radial pressure profiles have been computed for the different models and flow geometries.

For the Pom-Pom Model, we encountered numerical instabilities of the shooting algorithm which are due to the non-monotonic shear stress vs shear rate predictions of this model. For the Pompon Model and the TCMM Model, there exists a monotonic shear rate vs shear stress dependency and the shooting algorithm computed laminar flow profiles over a wide range of wall shear rates.

For the Pompon Model, we found an increase of the volumetric flow rate through tubes and concentric annuli for increasing number of Pompon arms. For the transversal normal stress in channel flow, the Pompon Model and the TCMM Model make qualitatively different predictions. Whereas the former gives positive transversal normal stresses, the latter gives negative transversal normal stresses on the channel walls. Furthermore, the Pompon Model predicts a non-monotonic behavior of the transversal normal 
Fig. 18 The same as in Fig. 17 for the corresponding extra stress tensor profiles



stresses near the mid-plane of the channel that is not found for the TCMM Model. An analogous behavior is found also for the radial stresses in annular flow.

In conclusion, we have solved coupled, multi-mode viscoelastic fluid models for polymer melts and polymer solutions to compute laminar flow and microstructural profiles in simple flow geometries such as channels, tubes, and concentric cylinders. The effect of coupled Couette and Poiseuille flow on the backbone stretch dynamics and the volumetric flow rate has been studied and the different viscoelastic fluid models have been compared with each other.

Acknowledgement MD acknowledges Prof. E. J. Windhab for providing the resources and encouragement necessary to carry out this type of research.

\section{Appendix}

In this appendix, the TCMM Model is solved for laminar Poiseuille flow through a circular tube of radius $R$. To accomplish this, we introduce cylindrical coordinates, $(r, \varphi, z)$. The tube wall is allowed to rotate with a constant angular velocity, $\Omega=U / R \mathbf{e}_{z}$. Then, the boundary conditions for the velocity field are

$v_{\varphi}(r=0)=0$,

$v_{\varphi}(r=R)=U$,

$v_{z}^{\prime}(r=0)=0$, 
$v_{z}(r=R)=0$,

and the cylinder axis is a symmetry line for the velocity and conformation tensor profiles. The TCMM equations in dimensionless form are given by Eqs. 45 and 46, where the cylinder radius, $R$, is the characteristic length of the flow problem.

For $\theta=0$, Eqs. 45 and 46 reduce to the 2MUCM model, which is solved analytically for laminar tube flow. For the azimuthal velocity, we get from Eq. 49, together with Eqs. 67 and 68,

$v_{\varphi}=-\frac{U}{R} r$

i.e., a constant angular shear rate in the tube. For the boundary conditions, Eqs. 69 and 70, we get from Eq. 53

$v_{z}=-\frac{1}{4 \widetilde{\eta}} \frac{\partial p}{\partial z}\left(R^{2}-r^{2}\right)$
The non-trivial components of the conformation tensors (Eq. 46) are

$$
\begin{aligned}
& C_{r r}^{i}=1, \quad C_{r \varphi}^{i}=0, \quad C_{r z}^{i}=\frac{\widetilde{\lambda}_{i}}{2 \widetilde{\eta}} \frac{\partial p}{\partial z} r \\
& C_{\varphi \varphi}^{i}=1, \quad C_{\varphi z}^{i}=0, \quad C_{z z}^{i}=2\left(\frac{\widetilde{\lambda}_{i}}{2 \widetilde{\eta}} \frac{\partial p}{\partial z} r\right)^{2}+1,
\end{aligned}
$$

for $i=\{1,2\}$. They are analogous to the channel flow solutions presented in the main body of the article. Note that for $\partial p / \partial z=0$ and $U \neq 0$, the 2MUCM Model gives $\mathbf{C}^{i}=1$ as the solution of the conformation tensor equations. The components of the extra stress tensor are calculated according to Eq. 9.

\section{References}

Alves MA, Pinho PJ, Oliveira FT (2001) Study of steady pipe and channel flows of a single-mode Phan Thien-Tanner fluid. J Non-Newton Fluid Mech 101:55-76

Beris AN, Edwards BJ (1994) Thermodynamics of flowing systems. Oxford Univ. Press, New York

Bird RB, Armstrong RC, Hassager O (1987) Dynamics of polymeric liquids: fluid mechanics, vol 1. Wiley, NY, USA

Chodankar CD, Schieber JD, Venerus DC (2003) Pom-Pom theory evaluation in double-step strain flows. J Rheol 47:413-427

Cruz DOA, Pinho FT (2004) Skewed Poiseuille-Couette flows of sPTT fluids in concentric annuli and channels. J Non-Newton Fluid Mech 121:1-14

Dressler M, Edwards BJ (2005) A method for calculating rheological and morphological properties of constant-volume polymer blend models in inhomogeneous shear fields. J Non-Newton Fluid Mech 130:77-95

Edwards BJ, Beris AN, Mavrantzas VG (1996) A model with two coupled Maxwell modes. J Rheol 40:917-942
Edwards BJ, Keffer DJ, Reneau CW (2002) An examination of the shear-thickening behavior of high molecular weight polymers dissolved in low-viscosity Newtonian solvents. J Appl Polym Sci 85:1714-1735

Giesekus H (1982) A simple constitutive equation for polymer fluids based on the concept of deformation-dependent tensorial mobility. J Non-Newton Fluid Mech 11:69-109

Jiang B, Keffer DJ, Edwards BJ, Allred JN (2003) Modeling shear thickening in dilute polymer solutions: temperature, concentration, and molecular weight dependencies. J Appl Polym Sci 90:2997-3011

Kamerkar PA, Edwards BJ, Keffer DJ, Reneau CW (2005) Shear thickening in dilute polymer solutions: transient analysis. Chem Eng Commun 192:89-107

McLeish TCB, Larson RG (1998) Molecular constitutive equations for a class of branched polymers: the Pom-Pom polymer. J Rheol 42:81-110

Oldroyd JG (1950) On the formulation of rheological equations of state. Proc R Soc London A 200:523-541

Oliveira PJ, Pinho FT (1999) Analytical solution for fully developed channel and pipe flow of Phan-Thien-Tanner fluids. J Fluid Mech 387:271-280

Öttinger HC (2001) Thermodynamic admissibility of the Pompon model for branched polymers. Rheol Acta 40:317-321
Phan-Thien NP, Tanner RI (1977) New constitutive equation derived from network theory. J Non-Newton Fluid Mech 2:353-365

Pinho FT, Oliveira PJ (2000a) Analysis of forced convection in pipes and channels with the simplified Phan-ThienTanner fluid. Int J Heat Mass Transfer 43:2273-2287

Pinho FT, Oliveira PJ (2000b) Axial annular flow of a nonlinear viscoelastic fluid - an analytical solution. J NonNewton Fluid Mech 93:325-337

Press WH, Teukolsky SA, Vetterling WT, BP Flannery (1992) Numerical recipes, 2nd edn. Cambridge Univ. Press, New York

Schleiniger G, Weinacht RJ (1991) Steady Poiseuille flows for a Giesekus fluid. J Non-Newton Fluid Mech 40:79-102

Tanner RI (2000) Engineering rheology, 2nd edn. Oxford engineering science series, number 52. Oxford Univ. Press, New York

Verbeeten WMH, Peters, GWM, Baaijens FPT (2001) Erratum: differential constitutive equations for polymer melts: the extended Pom-Pom model (2001). J Rheol 45(4):823-843, 1489 